\title{
Optimal Convergence Rates for the Strong Solutions to the Compressible MHD Equations with Potential Force
}

\author{
Miao Ouyang (iD \\ Department of Mathematics, Xiamen University of Technology, Xiamen 361012, China \\ Correspondence should be addressed to Miao Ouyang; mouyang@xmut.edu.cn
}

Received 15 October 2018; Accepted 19 March 2019; Published 21 April 2019

Academic Editor: Efstratios Tzirtzilakis

Copyright (c) 2019 Miao Ouyang. This is an open access article distributed under the Creative Commons Attribution License, which permits unrestricted use, distribution, and reproduction in any medium, provided the original work is properly cited.

In this paper, the large-time behavior of solutions to the Cauchy problem for the $3 \mathrm{D}$ compressible MHD equations is considered with the effect of external force. We construct the global unique solution with the small initial data near the stationary profile. The optimal $L^{p}-L^{2}(1 \leq p \leq 2)$ time decay rates of the solution to the system are built in multifrequency decompositions.

\section{Introduction}

Consider the following Cauchy problem of the full compressible MHD equations affected by the external potential force in $\mathbb{R}^{3}$ :

$$
\begin{aligned}
& \rho_{t}+\nabla \cdot(\rho u)=0, \\
& \rho\left[u_{t}+(u \cdot \nabla) u\right]+\nabla P(\rho, \theta) \\
& \quad=\mu \Delta u+(\mu+\lambda) \nabla(\nabla \cdot u)+(\nabla \times b) \times b+\rho \mathrm{F}, \\
& b_{t}-\nabla \times(u \times b)=-\nabla \times(\nabla \times(\nu b)), \\
& \rho c_{v}\left[\theta_{t}+(u \cdot \nabla) \theta\right]+\theta \mathrm{P}_{\theta}(\rho, \theta) \nabla \cdot u=\kappa \Delta \theta+\Psi[u], \\
& \nabla \cdot b=0
\end{aligned}
$$

where

$$
\begin{gathered}
(\rho, u, b, \theta)(0, x)=\left(\rho_{0}, u_{0}, b_{0}, \theta_{0}\right)(x) \longrightarrow \\
\left(\rho_{\infty}, 0,0, \theta_{\infty}\right),
\end{gathered}
$$

$$
\text { as }|x| \longrightarrow \infty
$$

Here $\rho \geq 0, u, b, \theta \in \mathbb{R}^{3}$, and $p(\rho, \theta)$ are density, velocity, magnetic field, temperature, and pressure, respectively. The constants $\mu, \lambda$ are the shear and bulk viscosity coefficients of the flow, satisfying the physical restrictions $\mu>0$ and $2 \mu+3 \lambda \geq 0$. The resistivity coefficient $\nu>0$ is inversely proportional to the electrical conductivity constant and acts as the magnetic diffusivity of magnetic fields. By the positive constant $\kappa$ we denote the heat conduction coefficient and $c_{v}>0$ is the specific heat at constant volume. In addition, $F=F(x)$ is an external force and $\Psi=\Psi[u]$ is the dissipation function:

$$
\Psi[u]=\frac{\mu}{2} \sum_{i, j=1}^{3}\left(\partial_{i} u_{j}+\partial_{j} u_{i}\right)^{2}+\lambda \sum_{j=1}^{3}\left(\partial_{j} u_{j}\right)^{2}
$$

It will be assumed that $P(\rho, \theta)$ is smooth in a neighborhood of $\left(\rho_{\infty}, \theta_{\infty}\right)$ with $P_{\rho}\left(\rho_{\infty}, \theta_{\infty}\right)>0$ and $P_{\theta}\left(\rho_{\infty}, \theta_{\infty}\right)>$ 0 throughout this paper. Both $\rho_{\infty}$ and $\theta_{\infty}$ are positive constants.

We only consider the potential force $F=-\nabla \Phi(x)$ in this work. Reference [1] has proofed the existence of the stationary solution to problem (1) and (2) under aforementioned assumptions. We give the solution $\left(\rho_{*}, u_{*}, b_{*}, \theta_{*}\right)$ in a neighborhood of $\left(\rho_{\infty}, 0,0, \theta_{\infty}\right)$ as

$$
\begin{aligned}
\int_{\rho_{\infty}}^{\rho_{*}(x)} \frac{P_{\rho}\left(\eta, \theta_{\infty}\right)}{\eta} d x+\Phi(x) & =0, \\
u_{*}(x) & =0, \\
b_{*}(x) & =0, \\
\theta_{*}(x) & =\theta_{\infty},
\end{aligned}
$$


and satisfy

$$
\begin{aligned}
& \left\|\rho_{*}-\rho_{\infty}\right\|_{H^{k}\left(\mathbb{R}^{3}\right)} \leq C\|\Phi\|_{H^{k}\left(\mathbb{R}^{3}\right)}, \quad 0 \leq k \leq 4 \\
& \sum_{k=1}^{4}\left\|(1+|x|) \nabla^{k}\left(\rho_{*}-\rho_{\infty}\right)\right\|_{L^{2}\left(\mathbb{R}^{3}\right)} \\
& \quad \leq C \sum_{k=1}^{4}\left\|(1+|x|) \nabla^{k} \Phi\right\|_{L^{2}\left(\mathbb{R}^{3}\right)} .
\end{aligned}
$$

The global unique solution is constructed to (1) near the steady state $\left(\rho_{*}, 0,0, \theta_{\infty}\right)$ when the initial perturbation belongs to the Sobolev space $H^{2}\left(\mathbb{R}^{3}\right)$. The main results are as follows.

Theorem 1. Suppose that $\left(\rho_{0}-\rho_{\infty}, u_{0}, b_{0}, \theta_{0}-\theta_{\infty}\right) \in H^{2}\left(\mathbb{R}^{3}\right)$, there exists constant $\varepsilon>0$ such that if

$$
\begin{gathered}
\left\|\left(\rho_{0}-\rho_{\infty}, u_{0}, b_{0}, \theta_{0}-\theta_{\infty}\right)\right\|_{H^{2}\left(\mathbb{R}^{3}\right)}+\|\Phi\|_{H^{4}\left(\mathbb{R}^{3}\right)} \\
+\sum_{k=1}^{4}\left\|(1+|x|) \nabla^{k} \Phi\right\|_{L^{2}\left(\mathbb{R}^{3}\right)} \leq \varepsilon
\end{gathered}
$$

then the initial value problem (1) and (2) admits a unique solution $(\rho, u, b, \theta)$ globally in time which satisfies

$$
\begin{aligned}
& \rho-\rho_{*} \in C\left([0, \infty) ; H^{2}\left(\mathbb{R}^{3}\right)\right) \\
& \cap C^{1}\left([0, \infty) ; H^{1}\left(\mathbb{R}^{3}\right)\right), \\
& u, b, \theta-\theta_{\infty} \in C\left([0, \infty) ; H^{2}\left(\mathbb{R}^{3}\right)\right) \\
& \cap C^{1}\left([0, \infty) ; L^{2}\left(\mathbb{R}^{3}\right)\right) .
\end{aligned}
$$

Moreover, if the initial data $\left(\rho_{0}-\rho_{\infty}, u_{0}, b_{0}, \theta_{0}-\theta_{\infty}\right)$ is bounded in $L^{p}\left(\mathbb{R}^{3}\right)$ for any given $1 \leq p \leq 2$, the solution $(\rho, u, b, \theta)$ satisfies the following decay-in-time estimates:

$$
\begin{aligned}
& \left\|\nabla\left(\rho-\rho_{*}, u, b, \theta-\theta_{\infty}\right)\right\|_{H^{1}\left(\mathbb{R}^{3}\right)} \\
& \leq C(1+t)^{-(3 / 2)(1 / p-1 / 2)} \quad \forall t \geq 0, \\
& \begin{array}{l}
\left\|\left(\rho-\rho_{*}, u, b, \theta-\theta_{\infty}\right)\right\|_{L^{q}\left(\mathbb{R}^{3}\right)} \leq C(1+t)^{-(3 / 2)(1 / p-1 / q)} \\
\| t \geq 0,2 \leq q \leq 6,
\end{array} \\
& \left\|\partial_{t}\left(\rho-\rho_{*}, u, b, \theta-\theta_{\infty}\right)\right\|_{L^{2}\left(\mathbb{R}^{3}\right)} \quad \forall t \geq 0, \\
& \leq C(1+t)^{-(3 / 2)(1 / p-1 / 2)} \quad \forall t \geq 0
\end{aligned}
$$

for some positive constant $C$.

Remark 2. In Theorem 1, using the Sobolev imbedding inequalities in Lemma 3, (7) together with (5) and (6) yields

$$
\begin{aligned}
& \left\|\rho-\rho_{*}\right\|_{H^{4}\left(\mathbb{R}^{3}\right)} \\
& \quad+\sum_{k=1}^{3}\left\|(1+|x|) \nabla^{k}\left(\rho_{*}-\rho_{\infty}\right)\right\|_{L^{2}\left(\mathbb{R}^{3}\right) \cap L^{3}\left(\mathbb{R}^{3}\right)} \leq C \varepsilon .
\end{aligned}
$$

Due to its importance in mathematics and physics, there are a lot of literatures devoted to the mathematical theory of the MHD fluid system, including the global existence, unique, and time decay rates of solutions with or without external forces; see [2-20] and references therein. For the initial value problem for three-dimensional isentropic MHD system ((1) with $\theta=$ const.), Li and Yu [13] obtained the global existence and the $L^{2}$ decay rate of classical solutions under the small oscillations on small initial data. Chen and Tan [4] obtained the decay estimate of solutions with the initial data in $L^{p}(p \in[1,6 / 5))$. Noting the special construction of (1), the authors in [18] obtained decay estimate of solutions when the initial data belongs to negative Sobolev space. For the initial boundary value problem for three-dimensional isentropic MHD system (1), we refer to [11]. Pu and Guo [17] extended the global existence and decay rate of solutions in $[4,13]$ to three-dimensional incompressible nonisentropic MHD system (1). For the initial boundary value for threedimensional nonisentropic MHD system, the global existence of weak solutions has been established in $[9,10]$.

If the magnetic field disappear $(b=0)$, system (1) deduces to Navier-Stokes equations. We only refer to time decay rates of solutions to the compressible Navier-Stokes equations. If there is no external force, Matsumura and Nishida [21] obtained the convergence rate for the compressible viscous and heat-conductive fluid in $\mathbb{R}^{3}$ :

$$
\left\|\left[\rho-\rho_{\infty}, u, \theta-\theta_{\infty}\right](t)\right\|_{2} \leq C(1+t)^{-3 / 4}, \quad t \geq 0 .
$$

For the same system, Ponce [22] gave the optimal $L^{p}$ convergence rate

$$
\begin{aligned}
& \left\|\nabla^{l}\left[\rho-\rho_{\infty}, u, \theta-\theta_{\infty}\right](t)\right\|_{L^{p}} \\
& \quad \leq C(1+t)^{-(n / 2)(1-1 / p)-l / 2}, \quad l=0,1,2, \quad t \geq 0,
\end{aligned}
$$

for the space dimension $n=2$ or 3 . When there is an external potential force $F=-\nabla \Phi(x)$, the first work to give explicit estimates for the decay rates for solutions to problem (1) and (2) was represented by Deckelnick [23]. In [23], when the initial perturbation only belongs to the Sobolev space $H^{3}(\Omega)$, the following decay estimates were established in an unbounded domain $\Omega \subset \mathbb{R}^{3}$ (the half space $\mathbb{R}_{+}^{3}=\left.x \in \mathbb{R}^{3}\right|_{x_{3}>0}$ or the exterior of a bounded domain with smooth boundary):

$$
\begin{gathered}
\left\|\nabla\left[\rho-\rho_{*}, u, \theta-\theta_{\infty}\right]\right\|_{L^{2}(\Omega)} \leq C(1+t)^{-1 / 4}, \\
\left\|\partial_{t}\left[\rho-\rho_{*}, u, \theta-\theta_{\infty}\right]\right\|_{L^{2}(\Omega)} \leq C(1+t)^{-1 / 2} .
\end{gathered}
$$

Duan et al. [24] obtained a similar result for the nonisentropic case

$$
\begin{gathered}
\left\|\nabla^{k}\left[\rho-\rho_{*}, u, \theta-\theta_{\infty}\right](t)\right\|_{L^{p}} \leq C(1+t)^{-(3 / 2)(1-1 / p)}, \\
2 \leq p \leq 6, \\
\left\|\left[\rho-\rho_{*}, u, \theta-\theta_{\infty}\right](t)\right\|_{L^{\infty}} \leq C(1+t)^{-5 / 4},
\end{gathered}
$$




$$
\begin{aligned}
\left\|\nabla\left[\rho-\rho_{\infty}, u, \theta-\theta_{\infty}\right](t)\right\|_{2} & \leq C(1+t)^{-5 / 4}, \\
\left\|\left[\rho_{t}, u_{t}, \theta_{t}\right](t)\right\| & \leq C(1+t)^{-5 / 4} .
\end{aligned}
$$

Recently, Wang [25] constructed the global unique solution near the stationary profile to the system for the small $H^{2}$ initial data and the optimal $L^{p}-L^{2}(1 \leq p \leq 2)$ time decay rates of the solution:

$$
\begin{aligned}
& \left\|\nabla\left[\rho-\rho_{*}, u, b, \theta-\theta_{\infty}\right]\right\|_{H^{1}\left(\mathbb{R}^{3}\right)} \\
& \quad \leq C(1+t)^{-(3 / 2)(1 / p-1 / 2)}, \\
& \left\|\left[\rho-\rho_{*}, u, b, \theta-\theta_{\infty}\right]\right\|_{L^{q}\left(\mathbb{R}^{3}\right)} \leq C(1+t)^{-(3 / 2)(1 / p-1 / q)}, \quad 2 \leq q \leq 6, \\
& \left\|\partial_{t}\left[\rho-\rho_{*}, u, b, \theta-\theta_{\infty}\right]\right\|_{L^{2}\left(\mathbb{R}^{3}\right)} \\
& \quad \leq C(1+t)^{-(3 / 2)(1 / p-1 / 2)} .
\end{aligned}
$$

In this paper, we will generalize the result in [25] to the three-dimensional magnetomicropolar fluid system (1). We will give the global existence and the optimal $L^{p}-L^{2}(1 \leq$ $p \leq 2)$ time decay rates of the solution. Notice that the first three equations in (1) have been studied in [25]. The magnetic field $b(t, x)$ satisfies the heat equation, which can be solved in Fourier space, $\widehat{b}=\exp \left(-|\xi|^{2} t\right) \widehat{b}_{0}$, whose decay estimate has been proved in many papers, for instance, [26, 27]. However, compared with the work in [25], we have to deal with the difficulties in the present paper caused by the strong coupling between the velocity vector field and the magnetic field, for instance, the influence of the nonlinear term $(\nabla \times H) \times H$ on the velocity vector field.

Notations. Denote $C$ as a generic positive constant. For multi-indices $\alpha=\left(\alpha_{1}, \alpha_{2}, \alpha_{3}\right)$ and $\beta=\left(\beta_{1}, \beta_{2}, \beta_{3}\right), C_{\alpha}^{\beta}=$ $\alpha ! /(\alpha-\beta) ! \beta !$ with $\beta \leq \alpha$ which means $\beta_{i} \leq \alpha_{i}$ for all $1 \leq i \leq n$. $\partial_{x}^{\alpha}=\partial_{x_{1}}^{\alpha_{1}} \partial_{x_{2}}^{\alpha_{2}} \partial_{x_{3}}^{\alpha_{3}}, \nabla=\left(\partial_{1}, \partial_{2}, \partial_{3}\right), \partial_{i}=\partial_{x_{i}}, i=1,2,3$, and $|\alpha|=\sum_{i=1}^{3} \alpha_{i}$. Denote $\nabla^{m}$ as a set composed of all $m$ th partial derivatives with respect to the variable $x . H^{m}\left(\mathbb{R}^{3}\right), m \in \mathbb{Z}_{+}$, denotes the usual Sobolev space with its norm

$$
\|f\|_{H^{m}\left(\mathbb{R}^{3}\right)} \triangleq \Sigma_{k=0}^{m}\left\|\nabla^{k} f\right\|_{L^{2}\left(\mathbb{R}^{3}\right)} .
$$

In particular, $\|\cdot\|_{m}$ means $\|\cdot\|_{H^{m}\left(\mathbb{R}^{3}\right)}$ and $\|\cdot\|_{m}$ means $\|\cdot\|_{L^{2}\left(\mathbb{R}^{3}\right)}$. As usual, $\langle\cdot, \cdot\rangle$ denotes the inner-product in $L^{2}\left(\mathbb{R}^{3}\right)$. The Fourier transform to the variable $x \in \mathbb{R}^{3}$ is applied by $\widehat{f}(\xi, t)=\int_{\mathbb{R}^{n}} f(x, t) e^{-\sqrt{-1} x \cdot \xi} d x$ and the inverse Fourier transform to the variable $\xi \in \mathbb{R}^{3}$ by $\left(\mathscr{F}^{-1} \widehat{f}(x, t)=\right.$ $(2 \pi)^{-n} \int_{\mathbb{R}^{3}} \widehat{f}(\xi, t) e^{\sqrt{-1} x \cdot \xi} d \xi$.

The outlet of paper is organized as follows. In Section 2, we reformulate the Cauchy problem (1)-(2) into a more suitable form. In Section 3, we establish the global existence of solutions to the problem in $H^{2}$-framework. Section 4 is devoted to the proof of the $L^{p}-L^{2}$ time decay rates of the solution and we complete the proof of Theorem 1 .

\section{Preliminary}

We are going to reformulate the Cauchy problem of the compressible MHD system (1) with the initial condition (2) and give some lemmas in this section.

Define

$$
\begin{aligned}
\widetilde{\rho}(x, t) & =\rho(x, t)-\rho_{*}(x), \\
\widetilde{u}(x, t) & =u(x, t), \\
\widetilde{b}(x, t) & =b(x, t), \\
\widetilde{\theta}(x, t) & =\theta(x, t)-\theta_{\infty}, \\
\bar{\rho}(x) & =\rho_{*}(x)-\rho_{\infty} .
\end{aligned}
$$

Then, by using (4), the MHD equations (1) are transformed as the following:

$$
\begin{aligned}
& \widetilde{\rho}_{t}+\rho_{\infty} \nabla \cdot \widetilde{u}=\widetilde{S}_{1}, \\
& \widetilde{u_{t}}-\frac{\mu}{\rho_{\infty}} \Delta \widetilde{u}-\frac{\mu+\lambda}{\rho_{\infty}} \nabla \nabla \cdot \widetilde{u}+\frac{P_{\rho}\left(\rho_{\infty}, \theta_{\infty}\right)}{\rho_{\infty}} \nabla \widetilde{\rho} \\
& \quad+\frac{P_{\theta}\left(\rho_{\infty}, \theta_{\infty}\right)}{\rho_{\infty}} \nabla \widetilde{\theta}=\widetilde{S}_{2}, \\
& \widetilde{b}_{t}-v \Delta \widetilde{b}=\widetilde{S}_{3}, \\
& \widetilde{\theta}_{t}-\frac{\kappa}{c_{v} \rho_{\infty}} \Delta \widetilde{\theta}+\frac{\theta_{\infty} P_{\theta}\left(\rho_{\infty}, \theta_{\infty}\right)}{c_{v} \rho_{\infty}} \nabla \cdot \widetilde{u}=\widetilde{S}_{4},
\end{aligned}
$$

and the initial condition (2) turns into

$$
\begin{gathered}
\left.(\widetilde{\rho}, \widetilde{u}, \widetilde{b}, \widetilde{\theta})(x, t)\right|_{t=0}=\left(\rho_{0}-\rho_{*}, u_{0}, b_{0}, \theta_{0}\right)(x) \longrightarrow \\
(0,0,0,0),
\end{gathered}
$$

as $|x| \longrightarrow \infty$,

where $\widetilde{S}_{1}, \widetilde{S}_{2}, \widetilde{S}_{3}$, and $\widetilde{S}_{4}$ are the source terms with

$$
\begin{aligned}
\widetilde{S}_{1} & =-\nabla \cdot(\tilde{\rho} \widetilde{u})-\nabla \cdot(\bar{\rho} \widetilde{u}), \\
\widetilde{S}_{2} & =-(\widetilde{u} \cdot \nabla) \widetilde{u}+\left[\frac{\mu}{\widetilde{\rho}+\rho_{*}}-\frac{\mu}{\rho_{\infty}}\right] \Delta \widetilde{u}+\left[\frac{\mu+\lambda}{\widetilde{\rho}+\rho_{*}}\right. \\
& \left.-\frac{\mu+\lambda}{\rho_{\infty}}\right] \nabla \nabla \cdot \widetilde{u}-\left[\frac{P_{\rho}\left(\widetilde{\rho}+\rho_{*}, \tilde{\theta}+\theta_{\infty}\right)}{\widetilde{\rho}+\rho_{*}}\right. \\
& \left.-\frac{P_{\rho}\left(\rho_{\infty}, \theta_{\infty}\right)}{\rho_{\infty}}\right] \nabla \widetilde{\rho}-\left[\frac{P_{\theta}\left(\widetilde{\rho}+\rho_{*}, \widetilde{\theta}+\theta_{\infty}\right)}{\widetilde{\rho}+\rho_{*}}\right. \\
& \left.-\frac{P_{\theta}\left(\rho_{\infty}, \theta_{\infty}\right)}{\rho_{\infty}}\right] \nabla \widetilde{\theta}-\left[\frac{P_{\rho}\left(\widetilde{\rho}+\rho_{*}, \widetilde{\theta}+\theta_{\infty}\right)}{\widetilde{\rho}+\rho_{*}}\right.
\end{aligned}
$$




$$
\begin{aligned}
& \left.-\frac{P_{\rho}\left(\rho_{*}, \theta_{\infty}\right)}{\rho_{*}}\right] \nabla \bar{\rho}+\left[\frac{1}{\widetilde{\rho}+\rho_{*}}-\frac{1}{\rho_{\infty}}\right][b \cdot \nabla b \\
& \left.-\frac{1}{2} \nabla|b|^{2}\right]+\frac{1}{\rho_{\infty}}\left[b \cdot \nabla b-\frac{1}{2} \nabla|b|^{2}\right],
\end{aligned}
$$

$$
\begin{aligned}
\widetilde{S}_{3} & =\widetilde{b} \cdot \nabla \widetilde{u}-\widetilde{b} \nabla \cdot \widetilde{u}-\widetilde{u} \cdot \nabla \widetilde{b}, \\
\widetilde{S}_{4} & =-(\widetilde{u} \cdot \nabla) \tilde{\theta}-\left[\frac{\left(\widetilde{\theta}+\theta_{\infty}\right) P_{\theta}\left(\widetilde{\rho}+\rho_{*}, \tilde{\theta}+\theta_{\infty}\right)}{c_{v}\left(\widetilde{\rho}+\rho_{*}\right)}\right. \\
& \left.-\frac{\theta_{\infty} P_{\infty}\left(\rho_{\infty}, \theta_{\infty}\right)}{c_{v} \rho_{\infty}}\right] \nabla \cdot \widetilde{u}+\left[\frac{\kappa}{c_{v}\left(\widetilde{\rho}+\rho_{*}\right)}-\frac{\kappa}{c_{v} \rho_{\infty}}\right] \\
& \cdot \Delta \widetilde{\theta}+\left[\frac{1}{c_{v}\left(\widetilde{\rho}+\rho_{*}\right)}-\frac{1}{c_{v} \rho_{\infty}}\right] \Psi[\widetilde{u}]+\frac{1}{c_{v} \rho_{\infty}} \Psi[\widetilde{u}] .
\end{aligned}
$$

For obtaining a symmetric system, we denote

$$
\begin{aligned}
\sigma(x, t) & =\widetilde{\rho}(x, t), \\
\omega(x, t) & =\sqrt{\frac{P_{1}}{\rho_{\infty}}} \widetilde{u}(x, t), \\
m(x, t) & =\sqrt{\frac{P_{1}}{\rho_{\infty}}} \widetilde{b}(x, t), \\
z(x, t) & =\sqrt{\frac{P_{2} \rho_{\infty}}{P_{1} P_{3}}} \widetilde{\theta}(x, t), \\
\mu_{1} & =\frac{\mu}{\rho_{\infty}}, \\
\mu_{2} & =\frac{\mu+\lambda}{\rho_{\infty}}, \\
\gamma_{1} & =\sqrt{P_{1} \rho_{\infty}}, \\
\gamma_{2} & =\sqrt{P_{2} P_{3}}, \\
\kappa_{1} & =\frac{\kappa}{c_{v}} \sqrt{\frac{P_{2}}{P_{1} P_{3} \rho_{\infty}}},
\end{aligned}
$$

with

$$
\begin{aligned}
& P_{1}=\frac{P_{\rho}\left(\rho_{\infty}, \theta_{\infty}\right)}{\rho_{\infty}}, \\
& P_{2}=\frac{P_{\theta}\left(\rho_{\infty}, \theta_{\infty}\right)}{\rho_{\infty}}, \\
& P_{3}=\frac{\theta_{\infty} P_{\theta}\left(\rho_{\infty}, \theta_{\infty}\right)}{c_{v} \rho_{\infty}} .
\end{aligned}
$$

Then (20) and (21) can be reformulated as

$$
\begin{aligned}
& \sigma_{t}+\gamma_{1} \nabla \cdot \omega=S_{1}, \\
& \omega_{t}-\mu_{1} \Delta \omega-\mu_{2} \nabla \nabla \cdot \omega+\gamma_{1} \nabla \sigma+\gamma_{2} \nabla z=S_{2}, \\
& m_{t}-\nu \Delta m=S_{3}, \\
& z_{t}-\kappa_{1} \Delta z+\gamma_{2} \nabla \cdot \omega=S_{4}, \\
& \left.(\sigma, \omega, m, z)(x, t)\right|_{t=0}=\left(\sigma_{0}, \omega_{0}, m_{0}, z_{0}\right)(x),
\end{aligned}
$$

where $S_{1}=\widetilde{S}_{1}, S_{2}=\sqrt{P_{1} / \rho_{\infty}} \widetilde{S}_{2}, S_{3}=\sqrt{P_{1} / \rho_{\infty}} \widetilde{S}_{3}, S_{4}=$ $\sqrt{P_{2} \rho_{\infty} / P_{1} P_{3}} \widetilde{S}_{4}$, and

$$
\begin{aligned}
& \left(\sigma_{0}, \omega_{0}, m_{0}, z_{0}\right)(x)=\left(\rho_{0}\right. \\
& \left.-\rho_{*}, \sqrt{\frac{\rho_{\infty}}{P_{1}}} u_{0}, \sqrt{\frac{\rho_{\infty}}{P_{1}}} b_{0}, \sqrt{\frac{P_{2} \rho_{\infty}}{P_{1} P_{3}}}\left(\theta_{0}-\theta_{\infty}\right)\right)(x) \longrightarrow
\end{aligned}
$$

$(0,0,0,0)$,

$$
\text { as }|x| \longrightarrow \infty \text {. }
$$

Then, we will focus on considering the global existence and time decay rates of the solution $(\rho, u, b, \theta)$ to the steady state $\left(\rho_{*}, 0,0, \theta_{\infty}\right)$, that is, the existence and decay rates of the perturbed solution $(\sigma, \omega, m, z)$ to problem (28). First of all, some inequalities are listed as follows for late use.

Lemma 3 (see [28]). Let $f \in H^{2}\left(\mathbb{R}^{3}\right)$. Then

(i) $\|f\|_{L^{\infty}} \leq C\|\nabla f\|^{1 / 2}\|\nabla f\|_{H^{1}}^{1 / 2} \leq C\|\nabla f\|_{H^{1}}$;

(ii) $\|f\|_{L^{6}} \leq C\|\nabla f\|$;

(iii) $\|f\|_{L^{q}} \leq C\|f\|_{H^{1}}, \quad 2 \leq q \leq 6$.

Lemma 4 (see [24]). Let $r_{1}, r_{2}>0$, then it holds that

$$
\begin{aligned}
& \int_{0}^{t}(1+t-\tau)^{-r_{1}}(1+\tau)^{-r_{2}} d \tau \\
& \quad \leq C\left(r_{1}, r_{2}\right)(1+t)^{-\min \left\{r_{1}, r_{2}, r_{1}+r_{2}-1-\eta\right\}},
\end{aligned}
$$

for an arbitrarily small $\eta>0$.

\section{Global Existence}

We will use the energy method to establish the global existence of solutions to the problem (28) in $H^{2}$-framework in this section. Let us define the solution space and the solution norm of the initial value problem (25) by

$$
\begin{aligned}
& X(0, T)=\left\{(\sigma, \omega, m, z) \mid \sigma \in C^{0}\left(0, T ; H^{2}\left(\mathbb{R}^{3}\right)\right)\right. \\
& \quad \cap C^{1}\left(0, T ; H^{1}\left(\mathbb{R}^{3}\right)\right), \omega, m, z \\
& \quad \in C^{0}\left(0, T ; H^{2}\left(\mathbb{R}^{3}\right)\right) \cap C^{1}\left(0, T ; L^{2}\left(\mathbb{R}^{3}\right)\right), \nabla \sigma \\
& \quad \in L^{2}\left(0, T ; H^{1}\left(\mathbb{R}^{3}\right)\right), \nabla(\omega, m, z) \\
& \left.\quad \in L^{2}\left(0, T ; H^{2}\left(\mathbb{R}^{3}\right)\right)\right\},
\end{aligned}
$$




$$
\begin{aligned}
& N(0, T)^{2}=\sup _{0 \leq t \leq T}\|(\sigma, \omega, m, z)(t)\|_{2}^{2} \\
& \quad+\int_{0}^{T}\left(\|\nabla \sigma(t)\|_{1}^{2}+\|\nabla(\omega, m, z)(t)\|_{2}^{2}\right) d t,
\end{aligned}
$$

where $T \in[0,+\infty]$. By the standard continuity argument, the global existence of solutions to (25) will be obtained from the combination of the local existence result with some a priori estimates.

Proposition 5 (local existence). Suppose that the initial data satisfy $\left(\sigma_{0}, \omega_{0}, m_{0}, z_{0}\right) \in H^{2}\left(\mathbb{R}^{3}\right)$ and (7). Then there exists a positive constant $T_{1}>0$ depending on $\left\|\left(\sigma_{0}, \omega_{0}, m_{0}, z_{0}\right)\right\|_{2}$, such that the initial value problem (25) has a unique solution $(\sigma, \omega, m, z) \in X\left(0, T_{1}\right)$ which satisfies $N\left(0, T_{1}\right) \leq 2 N(0,0)$.

Proof. The proof can be stated by using the standard iteration arguments. Refer to [2], for instance.

Proposition 6 (a priori estimates). Suppose that the initial value problem (28) has a solution $(\sigma, \omega, m, z) \in X(0, T)$, where $T$ is a positive constant. Under the assumptions of Theorem 1 , there exist a positive constant $C_{0}$ and a small positive constant $\delta$ which are independent of $T$, such that if

$$
\sup _{0 \leq t \leq T}\|(\sigma, \omega, m, z)(t)\|_{2} \leq \delta,
$$

where $\delta>\max \left\{\varepsilon, \sqrt{C_{0}} \varepsilon\right\}$ and $\varepsilon$ is given by (7), then it holds that, for any $t \in[0, T]$,

$$
\begin{aligned}
& \|(\sigma, \omega, m, z)(t)\|_{2}^{2} \\
& \quad+\int_{0}^{t}\left(\|\nabla \sigma(\tau)\|_{1}^{2}+\|\nabla(\omega, m, z)(\tau)\|_{2}^{2}\right) d \tau \\
& \quad \leq C_{0}\left\|\left(\sigma_{0}, \omega_{0}, m_{0}, z_{0}\right)\right\|_{2}^{2}<\delta^{2},
\end{aligned}
$$

where $C_{0}$ is independent of $\delta$.

Remark 7. The global existence and uniqueness of the solutions stated in Theorem 1 follow from Propositions 5 and 6. By (12), (33), and the Sobolev inequality, we have

$$
\frac{1}{2} \rho_{\infty} \leq \sigma+\rho_{*} \leq 2 \rho_{\infty}
$$

This will be used in following statement. Before proving Proposition 6, we supply Lemmas 8 and 9.

Lemma 8. For $0 \leq k \leq 2$, it holds that

$$
\begin{gathered}
\frac{1}{2} \frac{d}{d t}\left\|\nabla^{k}(\sigma, \omega, m, z)(t)\right\|^{2}+\frac{\mu_{1}}{2}\left\|\nabla^{k} \nabla \omega(t)\right\|^{2} \\
+\frac{\mu_{2}}{2}\left\|\nabla^{k} \nabla \cdot \omega(t)\right\|^{2}+\frac{\nu}{2}\left\|\nabla^{k} \nabla m(t)\right\|^{2} \\
+\frac{k_{1}}{2}\left\|\nabla^{k+1} z(t)\right\|^{2} \\
\leq C \delta\left\{\|\nabla \sigma(t)\|_{1}^{2}+\|\nabla(\omega, m, z)(t)\|_{2}^{2}\right\},
\end{gathered}
$$

for any $0 \leq t \leq T$.
Proof. For each multi-index $\alpha$ with $|\alpha|=k$, operating $\partial_{x}^{\alpha}$ to the first 4 equations of (28), then multiplying the resulting equations by $\partial_{x}^{\alpha} \sigma, \partial_{x}^{\alpha} \omega, \partial_{x}^{\alpha} m$, and $\partial_{x}^{\alpha} z$, respectively, summing up, and then integrating the result over $\mathbb{R}^{3}$, we obtain

$$
\begin{aligned}
& \frac{1}{2} \frac{d}{d t}\left\|\partial_{x}^{\alpha}(\sigma, \omega, m, z)(t)\right\|^{2}+\mu_{1}\left\|\nabla \partial_{x}^{\alpha} \omega(t)\right\|^{2} \\
& \quad+\mu_{2}\left\|\nabla \cdot \partial_{x}^{\alpha} \omega(t)\right\|^{2}+v\left\|\nabla \partial_{x}^{\alpha} m(t)\right\|^{2} \\
& \quad+\kappa_{1}\left\|\nabla \partial_{x}^{\alpha} z(t)\right\|^{2}=\left\langle\partial_{x}^{\alpha} \sigma(t), \partial_{x}^{\alpha} S_{1}(t)\right\rangle \\
& \quad+\left\langle\partial_{x}^{\alpha} \omega(t), \partial_{x}^{\alpha} S_{2}(t)\right\rangle+\left\langle\partial_{x}^{\alpha} m(t), \partial_{x}^{\alpha} S_{3}(t)\right\rangle \\
& \quad+\left\langle\partial_{x}^{\alpha} z(t), \partial_{x}^{\alpha} S_{4}(t)\right\rangle \triangleq I_{1}^{k}(t)+I_{2}^{k}(t)+I_{3}^{k}(t) \\
& \quad+I_{4}^{k}(t),
\end{aligned}
$$

where the nonlinear terms in (22)-(25) have the following equivalence properties:

$$
\begin{aligned}
& S_{1} \sim \partial_{i} \sigma \omega_{i}+\sigma \partial_{i} \omega_{i}+\partial_{i} \bar{\rho} \omega_{i}+\bar{\rho} \partial_{i} \omega_{i} \\
& S_{2} \sim \omega_{i} \partial_{i} \omega_{j}+\sigma \partial_{i} \partial_{i} \omega_{j}+\sigma \partial_{j} \partial_{i} \omega_{i}+\sigma \partial_{j} \sigma+z \partial_{j} \sigma \\
& \quad+\sigma \partial_{j} z+z \partial_{j} z+\bar{\rho} \partial_{i} \partial_{i} \omega_{j}+\bar{\rho} \partial_{j} \partial_{i} \omega_{i}+\bar{\rho} \partial_{j} \sigma \\
& \quad+\sigma \partial_{j} \bar{\rho}+z \partial_{j} \bar{\rho}+\bar{\rho} \partial_{j} z \\
& \quad+(\sigma+\bar{\rho}+1)\left(m_{i} \partial_{i} m_{j}+m_{j} \partial_{i} m_{j}\right), \\
& \quad S_{3} \sim \omega_{i} \partial_{i} m_{j}+m_{i} \partial_{i} \omega_{j}+m_{j} \partial_{i} \omega_{i}, \\
& S_{4} \sim \omega_{i} \partial_{i} z+\sigma \partial_{i} \partial_{i} z+\sigma \partial_{i} \omega_{i}+z \partial_{i} \omega_{i}+\sigma \Psi[\omega]+\Psi[\omega] \\
& \quad+\bar{\rho} \partial_{i} \partial_{i} z+\bar{\rho} \partial_{i} \omega_{i}+\bar{\rho} \Psi[\omega] .
\end{aligned}
$$

When $k=0$, by using the Hölder inequality, Lemma 3, (12), the a priori assumption (33), and the Young inequality, we obtain

$$
\begin{aligned}
& I_{1}^{0}(t) \leq C\left\{\left|\left\langle\sigma(t),\left(\partial_{i} \sigma \omega_{i}\right)(t)\right\rangle\right|\right. \\
& +\left|\left\langle\sigma(t),\left(\sigma \partial_{i} \omega_{i}\right)(t)\right\rangle\right|+\left|\left\langle\sigma(t),\left(\partial_{i} \bar{\rho} \omega_{i}\right)(t)\right\rangle\right| \\
& \left.+\left|\left\langle\sigma(t),\left(\bar{\rho} \partial_{i} \omega_{i}\right)(t)\right\rangle\right|\right\} \leq C\left\{\|\sigma\|_{L^{6}}\left\|\partial_{i} \sigma\right\|\left\|\omega_{i}\right\|_{L^{3}}\right. \\
& +\|\sigma\|_{L^{6}}\|\sigma\|_{L^{3}}\left\|\partial_{i} \omega\right\| \\
& +\left\|\frac{\sigma}{1+|x|}\right\|\left\|(1+|x|) \partial_{i} \bar{\rho}\right\|_{L^{3}}\left\|\omega_{i}\right\|_{L^{6}} \\
& \left.+\|\sigma\|_{L^{6}}\|\bar{\rho}\|_{L^{3}}\left\|\partial_{i} \omega_{i}\right\|\right\} \leq C\left\{\|\nabla \sigma\|^{2}\|\omega\|_{1}\right. \\
& +\|\nabla \sigma\|\|\sigma\|_{1}\|\nabla \omega\|+\|\nabla \sigma\|\left\|(1+|x|) \partial_{i} \bar{\rho}\right\|_{1}\|\nabla \omega\| \\
& \left.+\|\nabla \sigma\|\|\bar{\rho}\|_{1}\|\nabla \omega\|\right\} \leq C \delta\left\{\|\nabla \sigma\|^{2}+\|\nabla \omega\|^{2}\right\},
\end{aligned}
$$

where we have used the Hardy inequality as follows:

$$
\left\|\frac{\sigma}{1+|x|}\right\| \leq\|\nabla \sigma\| .
$$


In a similar way, we get

$$
\begin{aligned}
& I_{2}^{0}(t) \leq C \delta\left\{\|\nabla \sigma\|^{2}+\|\nabla \omega\|_{1}^{2}+\|\nabla m\|^{2}+\|\nabla z\|^{2}\right\}, \\
& I_{3}^{0}(t) \leq C \delta\left\{\|\nabla \omega\|^{2}+\|\nabla m\|^{2}\right\}, \\
& I_{4}^{0}(t) \leq C \delta\left\{\|\nabla z\|_{1}^{2}+\|\nabla \omega\|^{2}\right\} .
\end{aligned}
$$

Putting (39), (41) into (37) and taking $\delta$ sufficiently small, we have

$$
\begin{aligned}
\frac{1}{2} \frac{d}{d t}\|(\sigma, \omega, m, z)(t)\|^{2}+\frac{\mu_{1}}{2}\|\nabla \omega(t)\|^{2} & \\
& +\frac{\mu_{2}}{2}\|\nabla \cdot \omega(t)\|^{2}+\frac{v}{2}\|\nabla m(t)\|^{2}+\frac{\kappa_{1}}{2}\|\nabla z(t)\|^{2} \\
\leq & C \delta\left\{\|\nabla \sigma(t)\|^{2}+\left\|\nabla^{2} \omega(t)\right\|^{2}+\left\|\nabla^{2} z(t)\right\|^{2}\right\} .
\end{aligned}
$$

When $1 \leq k \leq 2$, the terms on the RHS of (37) can be estimated as follows. For $I_{1}^{k}(t)$, from (38), we get

$$
\begin{aligned}
& I_{1}^{k}(t) \leq C\left\{\left|\left\langle\partial_{x}^{\alpha} \sigma(t), \partial_{x}^{\alpha}\left(\partial_{i} \sigma \omega_{i}\right)(t)\right\rangle\right|\right. \\
& +\left|\left\langle\partial_{x}^{\alpha} \sigma(t), \partial_{x}^{\alpha}\left(\sigma \partial_{i} \omega_{i}\right)(t)\right\rangle\right| \\
& +\left|\left\langle\partial_{x}^{\alpha} \sigma(t), \partial_{x}^{\alpha}\left(\partial_{i} \bar{\rho} \omega_{i}\right)(t)\right\rangle\right| \\
& \left.+\left|\left\langle\partial_{x}^{\alpha} \sigma(t), \partial_{x}^{\alpha}\left(\bar{\rho} \partial_{i} \omega_{i}\right)(t)\right\rangle\right|\right\} .
\end{aligned}
$$

For the first term on the RHS of (43), by using integration by parts, the Hölder inequality, the Young inequality, Lemma 3, and the a priori assumption (33), we have

$$
\begin{aligned}
\left|\left\langle\partial_{x}^{\alpha} \sigma(t), \partial_{x}^{\alpha}\left(\partial_{i} \sigma \omega_{i}\right)(t)\right\rangle\right| & \\
\leq & C\left|\left\langle\partial_{x}^{\alpha} \sigma, \partial_{x}^{\alpha} \partial_{i} \sigma \omega_{i}\right\rangle\right| \\
& +C \sum_{\beta \leq \alpha,|\beta| \leq|\alpha|-1} C_{\alpha}^{\beta}\left|\left\langle\partial_{x}^{\alpha} \sigma, \partial_{x}^{\beta} \partial_{i} \sigma \partial_{x}^{\alpha-\beta} \omega_{i}\right\rangle\right| \\
= & \frac{C}{2}\left|\left\langle\left(\partial_{x}^{\alpha} \sigma\right)^{2}, \partial_{i} \omega_{i}\right\rangle\right| \\
& +C\left\{\sum_{|\beta|=0}+\sum_{1 \leq|\beta| \leq|\alpha|-1}\right\}\left|\left\langle\partial_{x}^{\alpha} \sigma, \partial_{x}^{\beta} \partial_{i} \sigma \partial_{x}^{\alpha-\beta} \omega_{i}\right\rangle\right| \\
\leq & C\left\|\partial_{i} \omega_{i}\right\|_{L^{\infty}}\left\|\partial_{x}^{\alpha} \sigma\right\|^{2}+C\left\|\partial_{x}^{\alpha} \sigma\right\|\left\|\partial_{i} \sigma\right\|_{L^{3}}\left\|\partial_{x}^{\alpha} \omega_{i}\right\|_{L^{6}} \\
& +\delta\left\|\partial_{x}^{\alpha} \sigma\right\|^{2}+\frac{C}{\delta} \sum_{1 \leq|\beta| \leq|\alpha|-1}\left\|\nabla \partial_{x}^{\alpha-\beta} \omega_{i}\right\|^{2}\left\|\partial_{x}^{\beta} \partial_{i} \sigma\right\|^{2} \\
\leq & C \delta\left\|\partial_{x}^{\alpha} \sigma\right\|\|\nabla \omega\|_{2}+C \delta\left\|\partial_{x}^{\alpha} \sigma\right\|\left\|\nabla \partial_{x}^{\alpha} \omega\right\|+\delta\left\|\partial_{x}^{\alpha} \sigma\right\|^{2} \\
& +C \delta\|\nabla \omega\|_{1}^{2} \leq C \delta\left\{\left\|\partial_{x}^{\alpha} \sigma\right\|^{2}+\|\nabla \omega\|_{2}^{2}\right\},
\end{aligned}
$$

where the terms including the sum of $\beta$ with $1 \leq|\beta| \leq|\alpha|-1$ will be vanished if $|\alpha|=1$. Similarly, we have

$$
\begin{aligned}
& \left|\left\langle\partial_{x}^{\alpha} \sigma(t), \partial_{x}^{\alpha}\left(\sigma \partial_{i} \omega_{i}\right)(t)\right\rangle\right| \\
& =\left\{\sum_{|\beta|=0}+\sum_{1 \leq|\beta| \leq|\alpha|-1}+\sum_{|\beta|=|\alpha|}\right\} C_{\alpha}^{\beta} \mid\left\langle\partial_{x}^{\alpha} \sigma,\right. \\
& \left.\partial_{x}^{\alpha-\beta} \sigma \partial_{x}^{\beta} \partial_{i} \omega_{i}\right\rangle \mid \leq C\left\|\partial_{i} \omega_{i}\right\|_{L^{\infty}}\left\|\partial_{x}^{\alpha} \sigma\right\|^{2} \\
& +C \sum_{1 \leq|\beta| \leq|\alpha|}\left\|\partial_{x}^{\alpha} \sigma\right\|\left\|\partial_{x}^{\alpha-\beta} \sigma\right\|_{L^{6}}\left\|\partial_{x}^{\beta} \partial_{i} \omega_{i}\right\|_{L^{3}}+C\left\|\partial_{x}^{\alpha} \sigma\right\| \\
& \cdot\|\sigma\|_{L^{\infty}}\left\|\partial_{x}^{\alpha} \partial_{i} \omega\right\| \leq C \delta\left\|\partial_{x}^{\alpha} \sigma\right\|\left\|\partial_{i} \omega_{i}\right\|_{2}+\delta\left\|\partial_{x}^{\alpha} \sigma\right\|^{2} \\
& +\frac{C}{\delta} \sum_{1 \leq|\beta| \leq|\alpha|-1}\left\|\partial_{x}^{\alpha-\beta} \nabla \sigma\right\|^{2}\left\|\partial_{x}^{\beta} \partial_{i} \omega_{i}\right\|_{1}^{2}+C \delta\left\|\partial_{x}^{\alpha} \sigma\right\| \\
& \cdot\left\|\partial_{x}^{\alpha} \partial_{i} \omega_{i}\right\| \leq C \delta\left\{\left\|\partial_{x}^{\alpha} \sigma\right\|^{2}+\|\nabla \omega\|_{2}^{2}\right\} .
\end{aligned}
$$

By the Hölder inequality, the Young inequality, Lemma 3, and (12), the third term on the RHS of (43) can be estimated as follows:

$$
\begin{aligned}
& \left|\left\langle\partial_{x}^{\alpha} \sigma(t), \partial_{x}^{\alpha}\left(\partial_{i} \bar{\rho} \omega_{i}\right)(t)\right\rangle\right| \\
& \quad \leq \sum_{|\beta| \leq|\alpha|}\left|\left\langle\partial_{x}^{\alpha} \sigma, \partial_{x}^{\beta} \partial_{i} \bar{\rho} \partial_{x}^{\alpha-\beta} \omega_{i}\right\rangle\right| \\
& \quad \leq C \sum_{|\beta| \leq|\alpha|}\left\|\partial_{x}^{\alpha} \sigma\right\|\left\|\partial_{x}^{\beta} \partial_{i} \bar{\rho}\right\|_{L^{3}}\left\|\partial_{x}^{\alpha-\beta} \omega_{i}\right\|_{L^{6}} \\
& \quad \leq \delta\left\|\partial_{x}^{\alpha} \sigma\right\|^{2}+\frac{C}{\delta} \sum_{|\beta| \leq|\alpha|}\left\|\partial_{x}^{\beta} \partial_{i} \bar{\rho}\right\|_{1}^{2}\left\|\partial_{x}^{\alpha-\beta} \nabla \omega_{i}\right\|^{2} \\
& \quad \leq C \delta\left\{\left\|\partial_{x}^{\alpha} \sigma\right\|^{2}+\|\nabla \omega\|_{2}^{2}\right\} .
\end{aligned}
$$

By a similar argument, the fourth term on the RHS of (43) has following estimations:

$$
\begin{aligned}
& \left|\left\langle\partial_{x}^{\alpha} \sigma(t), \partial_{x}^{\alpha}\left(\bar{\rho} \partial_{i} \omega_{i}\right)(t)\right\rangle\right| \\
& =\left\{\sum_{|\beta|=0}+\sum_{1 \leq|\beta| \leq|\alpha|}\right\}\left|\left\langle\partial_{x}^{\alpha} \sigma, \partial_{x}^{\beta} \bar{\rho} \partial_{x}^{\alpha-\beta} \partial_{i} \omega_{i}\right\rangle\right| \\
& \leq C\left\|\partial_{x}^{\alpha} \sigma\right\|\|\bar{\rho}\|_{L^{\infty}}\left\|\partial_{x}^{\alpha} \partial_{i} \omega_{i}\right\| \\
& \quad+C \sum_{1 \leq|\beta| \leq|\alpha|}\left\|\partial_{x}^{\alpha} \sigma\right\|\left\|\partial_{x}^{\beta} \bar{\rho}\right\|_{L^{3}}\left\|\partial_{x}^{\alpha-\beta} \partial_{i} \omega_{i}\right\|_{L^{6}} \\
& \leq C \delta\left\{\left\|\partial_{x}^{\alpha} \sigma\right\|^{2}+\|\nabla \omega\|_{2}^{2}\right\} .
\end{aligned}
$$

Therefore, putting (44)-(47) into (43) yields

$$
I_{1}^{k}(t) \leq C \delta\left\{\left\|\partial_{x}^{\alpha} \sigma\right\|^{2}+\|\nabla \omega\|_{2}^{2}\right\}
$$


For $I_{2}^{k}(t)$, from (38), it is obvious that $I_{2}^{k}(t)$

$$
\begin{aligned}
\leq & \left|\left\langle\partial_{x}^{\alpha} \omega, \partial_{x}^{\alpha}\left(\omega_{i} \partial_{i} \omega_{j}\right)\right\rangle\right|+\left|\left\langle\partial_{x}^{\alpha} \omega, \partial_{x}^{\alpha}\left(\sigma \partial_{i} \partial_{i} \omega_{j}\right)\right\rangle\right| \\
& +\left|\left\langle\partial_{x}^{\alpha} \omega, \partial_{x}^{\alpha}\left(\sigma \partial_{j} \partial_{i} \omega_{i}\right)\right\rangle\right|+\left|\left\langle\partial_{x}^{\alpha} \omega, \partial_{x}^{\alpha}\left(\sigma \partial_{j} \sigma\right)\right\rangle\right| \\
& +\left|\left\langle\partial_{x}^{\alpha} \omega, \partial_{x}^{\alpha}\left(z \partial_{j} \sigma\right)\right\rangle\right|+\left|\left\langle\partial_{x}^{\alpha} \omega, \partial_{x}^{\alpha}\left(\sigma \partial_{j} z\right)\right\rangle\right| \\
& +\left|\left\langle\partial_{x}^{\alpha} \omega, \partial_{x}^{\alpha}\left(z \partial_{j} z\right)\right\rangle\right|+\left|\left\langle\partial_{x}^{\alpha} \omega, \partial_{x}^{\alpha}\left(\bar{\rho} \partial_{i} \partial_{i} \omega_{j}\right)\right\rangle\right| \\
& +\left|\left\langle\partial_{x}^{\alpha} \omega, \partial_{x}^{\alpha}\left(\bar{\rho} \partial_{j} \partial_{i} \omega_{i}\right)\right\rangle\right|+\left|\left\langle\partial_{x}^{\alpha} \omega, \partial_{x}^{\alpha}\left(\bar{\rho} \partial_{j} \sigma\right)\right\rangle\right| \\
& +\left|\left\langle\partial_{x}^{\alpha} \omega, \partial_{x}^{\alpha}\left(\sigma \partial_{j} \bar{\rho}\right)\right\rangle\right|+\left|\left\langle\partial_{x}^{\alpha} \omega, \partial_{x}^{\alpha}\left(z \partial_{j} \bar{\rho}\right)\right\rangle\right| \\
& +\left|\left\langle\partial_{x}^{\alpha} \omega, \partial_{x}^{\alpha}\left(\bar{\rho} \partial_{j} z\right)\right\rangle\right| \\
& +\left|\left\langle\partial_{x}^{\alpha} \omega, \partial_{x}^{\alpha}\left((\sigma+\bar{\rho}+1)\left(m_{j} \partial_{i} m_{j}+m_{i} \partial_{i} m_{i}\right)\right)\right\rangle\right| .
\end{aligned}
$$

For the second term on the RHS of (49), based on integration by parts, the Hölder inequality, Lemma 3, and (33), we have

$$
\begin{aligned}
\left|\left\langle\partial_{x}^{\alpha} \omega(t), \partial_{x}^{\alpha}\left(\sigma \partial_{i} \partial_{i} \omega_{j}\right)\right\rangle\right| & \left\{\sum_{|\beta|=0}+\sum_{1 \leq|\beta| \leq|\alpha|}\right\}\left|\left\langle\partial_{x}^{\alpha} \omega, \partial_{x}^{\beta} \sigma \partial_{x}^{\alpha-\beta} \partial_{i} \partial_{i} \omega_{j}\right\rangle\right| \\
\leq & \left|\left\langle\left(\partial_{x}^{\alpha} \partial_{i} \omega\right)^{2}, \sigma\right\rangle\right|+\left|\left\langle\partial_{x}^{\alpha} \omega, \partial_{i} \sigma \partial_{x}^{\alpha} \partial_{i} \omega_{j}\right\rangle\right| \\
& +\sum_{1 \leq|\beta| \leq|\alpha|-1}\left|\left\langle\partial_{x}^{\alpha} \omega, \partial_{x}^{\beta} \sigma \partial_{x}^{\alpha-\beta} \partial_{i} \partial_{i} \omega_{j}\right\rangle\right| \\
& +\left|\left\langle\partial_{x}^{\alpha} \omega, \partial_{x}^{\alpha} \sigma \partial_{i} \partial_{i} \omega_{j}\right\rangle\right| \\
\leq & C\|\sigma\|_{L^{\infty}}\left\|\partial_{x}^{\alpha} \partial_{i} \omega\right\|^{2}+\left\|\partial_{x}^{\alpha} \omega\right\|_{L^{6}}\left\|\partial_{i} \sigma\right\|_{L^{3}}\left\|\partial_{x}^{\alpha} \partial_{i} \omega_{j}\right\| \\
& +\sum_{1 \leq|\beta| \leq|\alpha|-1}\left\|\partial_{x}^{\alpha} \omega\right\|_{L^{6}}\left\|\partial_{x}^{\beta} \sigma\right\|_{L^{3}}\left\|\partial_{x}^{\alpha-\beta} \partial_{i} \partial_{i} \omega_{j}\right\| \\
& +\left\|\partial_{x}^{\alpha} \omega\right\|_{L^{6}}\left\|\partial_{x}^{\alpha} \sigma\right\|\left\|\partial_{i} \partial_{i} \omega_{j}\right\|_{L^{3}} \\
\leq & C \delta\left\{\left\|\partial_{x}^{\alpha} \nabla \omega\right\|^{2}+\left\|\nabla^{2} \omega\right\|_{1}^{2}\right\} .
\end{aligned}
$$

For the 11th term on the RHS of (49), by using the Hölder inequality, the Hardy inequality, Lemma 3, and (12), we obtain

$$
\begin{aligned}
& \left|\left\langle\partial_{x}^{\alpha} \omega(t), \partial_{x}^{\alpha}\left(\sigma \partial_{j} \bar{\rho}\right)\right\rangle\right| \\
& \quad=\left\{\sum_{|\beta|=0}+\sum_{1 \leq|\beta| \leq|\alpha|}\right\}\left|\left\langle\partial_{x}^{\alpha} \omega(t), \partial_{x}^{\beta} \sigma \partial_{x}^{\alpha-\beta} \partial_{j} \bar{\rho}\right\rangle\right| \\
& \quad \leq C\left\|\partial_{x}^{\alpha} \omega\right\|_{L^{6}}\left\|(1+|x|) \partial_{x}^{\alpha} \partial_{j} \bar{\rho}\right\|_{L^{3}}\left\|\frac{\sigma}{1+|x|}\right\|
\end{aligned}
$$

$+\sum_{1 \leq|\beta| \leq|\alpha|}\left\|\partial_{x}^{\alpha} \omega\right\|_{L^{6}}\left\|\partial_{x}^{\beta} \sigma\right\|\left\|\partial_{x}^{\alpha-\beta} \partial_{j} \bar{\rho}\right\|_{L^{3}} \leq C \delta\left\|\partial_{x}^{\alpha} \nabla \omega\right\|$

$$
\begin{aligned}
& \cdot\|\nabla \sigma\|+C \delta \sum_{1 \leq|\beta| \leq|\alpha|}\left\|\partial_{x}^{\alpha} \nabla \omega\right\|\left\|\partial_{x}^{\beta} \sigma\right\| \leq C \delta\left\{\left\|\partial_{x}^{\alpha} \nabla \omega\right\|^{2}\right. \\
& \left.+\|\nabla \sigma\|_{1}^{2}\right\}, \\
& \left|\left\langle\partial_{x}^{\alpha} \omega(t), \partial_{x}^{\alpha}\left(\sigma m_{j} \partial_{i} m_{j}\right)\right\rangle\right| \\
& =\left\{\sum_{|\beta|=0}+\sum_{1 \leq|\beta| \leq|\alpha|}\right\} \mid\left\langle\partial_{x}^{\alpha} \omega(t),\right. \\
& \left.\partial_{x}^{\beta} \sigma \partial_{x}^{\alpha-\beta}\left(\sigma m_{j} \partial_{i} m_{j}\right)\right\rangle \mid \leq C\|\sigma\|_{L^{\infty}}\left\|\partial_{x}^{\alpha}\left(m_{j} \partial_{i} m_{j}\right)\right\| \\
& \cdot\left\|\partial_{x}^{\alpha} \omega(t)\right\|+\sum_{1 \leq|\beta| \leq|\alpha|}\left\|\partial_{x}^{\alpha} \omega\right\|_{L^{6}}\left\|\partial_{x}^{\beta} \sigma\right\| \\
& \cdot\left\|\partial_{x}^{\alpha-\beta}\left(m_{j} \partial_{i} m_{j}\right)\right\| L_{L^{3}} \leq C\|\sigma\|_{L^{\infty}}\|m\|_{L^{\infty}}\left\|\partial_{x}^{\alpha} \omega\right\| \| \partial_{x}^{\alpha} \\
& \nabla m\left\|+\sum_{1 \leq|\beta| \leq|\alpha|}\right\| \partial_{x}^{\alpha} \nabla \omega\|\| m\left\|_{L^{6}}\right\| \partial_{x}^{\beta} \sigma\|\| \partial_{x}^{\alpha-\beta} \nabla m \|_{L^{6}} \\
& \quad \leq C \delta\left\{\left\|\partial_{x}^{\alpha} \nabla \omega\right\|^{2}+\left\|\partial_{x}^{\alpha} \nabla m\right\|^{2}\right\},
\end{aligned}
$$

where we used (33). Similarly,

$$
\begin{array}{r}
\left|\left\langle\partial_{x}^{\alpha} \omega(t), \partial_{x}^{\alpha}\left(\sigma m_{i} \partial_{i} m_{j}\right)\right\rangle\right| \\
\leq C \delta\left\{\left\|\partial_{x}^{\alpha} \nabla \omega\right\|^{2}+\left\|\partial_{x}^{\alpha} \nabla m\right\|^{2}\right\}, \\
\left|\left\langle\partial_{x}^{\alpha} \omega, \partial_{x}^{\alpha}\left(m_{j} \partial_{i} m_{j}+m_{i} \partial_{i} m_{i}\right)\right\rangle\right| \\
\leq C \delta\left\{\left\|\partial_{x}^{\alpha} \nabla \omega\right\|^{2}+\left\|\partial_{x}^{\alpha} \nabla m\right\|^{2}\right\} .
\end{array}
$$

The other terms on the RHS of (49) can be estimated in some similar way.

$$
\begin{aligned}
I_{2}^{k}(t) & \leq C \delta\left\{\|\nabla \sigma\|_{1}^{2}+\left\|\partial_{x}^{\alpha} \omega\right\|^{2}+\left\|\partial_{x}^{\alpha} \nabla \omega\right\|^{2}+\left\|\partial_{x}^{\alpha} \nabla m\right\|^{2}\right. \\
+ & \left.\|\nabla \omega\|_{2}^{2}+\|\nabla z\|_{1}^{2}\right\} .
\end{aligned}
$$

Now, let us estimate $I_{3}^{k}(t), I_{4}^{k}(t)$. Similar to the estimate before, $I_{3}^{k}(t)$ and $I_{3}^{k}(t)$ hold that

$$
\begin{aligned}
& I_{3}^{k}(t) \leq C \delta\left\{\left\|\partial_{x}^{\alpha} m\right\|^{2}+\left\|\partial_{x}^{\alpha} \nabla m\right\|^{2}\right\}, \\
& I_{4}^{k}(t) \leq C \delta\left\{\left\|\partial_{x}^{\alpha} z\right\|^{2}+\left\|\partial_{x}^{\alpha} \nabla z\right\|^{2}+\|\nabla \omega\|_{2}^{2}+\|\nabla z\|_{2}^{2}\right\} .
\end{aligned}
$$

Thus (37) together with (48), (53), (54), and (55) yields

$$
\begin{aligned}
\frac{1}{2} \frac{d}{d t}\left\|\partial_{x}^{\alpha}(\sigma, \omega, m, z)(t)\right\|^{2}+\frac{\mu_{1}}{2}\left\|\nabla \partial_{x}^{\alpha} \omega(t)\right\|^{2} \\
+\frac{\mu_{2}}{2}\left\|\nabla \cdot \partial_{x}^{\alpha} \omega(t)\right\|^{2}+\frac{v}{2}\left\|\nabla \partial_{x}^{\alpha} m(t)\right\|^{2} \\
+\frac{\kappa_{1}}{2}\left\|\nabla \partial_{x}^{\alpha} z(t)\right\|^{2}
\end{aligned}
$$




$$
\begin{aligned}
\leq & C \delta\left\{\left\|\partial_{x}^{\alpha}(\sigma, \omega, m, z)\right\|^{2}+\left\|\partial_{x}^{\alpha} \nabla(\omega, m, z)\right\|^{2}\right. \\
& +\|\nabla \sigma\|_{1}^{2}+\|\nabla(\omega, m, z)\|_{2}^{2},
\end{aligned}
$$

where we have taken $\delta>0$ sufficiently small. Therefore, (42) and (56) give (36).

Now, the following lemma will give the dissipative estimates for $\nabla \sigma$.

Lemma 9. For $0 \leq k \leq 1$, it holds that

$$
\begin{aligned}
\frac{d}{d t}\left\langle\nabla^{k} \omega(t), \nabla^{k} \nabla \sigma(t)\right\rangle+\frac{\gamma_{1}}{2}\left\|\nabla^{k} \nabla \sigma(t)\right\|^{2} \\
\leq C\left\{\left\|\nabla^{k} \nabla \omega(t)\right\|_{1}^{2}+\left\|\nabla^{k} \nabla z(t)\right\|^{2}\right\} \\
+C \delta\left\{\|\nabla \sigma(t)\|_{1}^{2}+\|\nabla(\omega, m, z)(t)\|_{2}^{2}\right\},
\end{aligned}
$$

for any $0 \leq t \leq T$.

Proof. From $(28)_{2}$, we get

$$
\gamma_{1} \nabla \sigma=-\omega_{t}+\mu_{1} \triangle \omega+\mu_{2} \nabla \nabla \cdot \omega-\gamma_{2} \nabla z+S_{2} .
$$

By applying $\partial_{x}^{\alpha}(|\alpha|=k)$ to (58), multiplying it by $\nabla \partial_{x}^{\alpha} \sigma$, and then integrating over $\mathbb{R}^{3}$, we have

$$
\begin{aligned}
\gamma_{1}\left\|\nabla \partial_{x}^{\alpha} \sigma\right\|^{2}= & -\left\langle\partial_{x}^{\alpha} \omega_{t}, \nabla \partial_{x}^{\alpha} \sigma\right\rangle+\mu_{1}\left\langle\partial_{x}^{\alpha} \Delta \omega, \nabla \partial_{x}^{\alpha} \sigma\right\rangle \\
& +\mu_{2}\left\langle\partial_{x}^{\alpha} \nabla \nabla \cdot \omega, \nabla \partial_{x}^{\alpha} \sigma\right\rangle \\
& -\gamma_{2}\left\langle\partial_{x}^{\alpha} \nabla z, \nabla \partial_{x}^{\alpha} \sigma\right\rangle+\left\langle\partial_{x}^{\alpha} S_{2}, \nabla \partial_{x}^{\alpha} \sigma\right\rangle .
\end{aligned}
$$

Using integration by parts and $(28)_{1}$, we obtain

$$
\begin{aligned}
-\left\langle\partial_{x}^{\alpha} \omega_{t}, \nabla \partial_{x}^{\alpha} \sigma\right\rangle= & -\frac{d}{d t}\left\langle\partial_{x}^{\alpha} \omega, \nabla \partial_{x}^{\alpha} \sigma\right\rangle+\left\langle\partial_{x}^{\alpha} \omega, \nabla \partial_{x}^{\alpha} \sigma_{t}\right\rangle \\
= & -\frac{d}{d t}\left\langle\partial_{x}^{\alpha} \omega, \nabla \partial_{x}^{\alpha} \sigma\right\rangle \\
& -\gamma_{1}\left\langle\partial_{x}^{\alpha} \omega, \nabla \partial_{x}^{\alpha} \nabla \cdot \omega\right\rangle \\
& +\left\langle\partial_{x}^{\alpha} \omega, \nabla \partial_{x}^{\alpha} S_{1}\right\rangle .
\end{aligned}
$$

Adding (59) and (60) gives

$$
\begin{aligned}
\gamma_{1}\left\|\nabla \partial_{x}^{\alpha} \sigma\right\|^{2}+\frac{d}{d t}\left\langle\partial_{x}^{\alpha} \omega, \nabla \partial_{x}^{\alpha} \sigma\right\rangle & \\
= & \mu_{1}\left\langle\partial_{x}^{\alpha} \Delta \omega, \nabla \partial_{x}^{\alpha} \sigma\right\rangle+\mu_{2}\left\langle\partial_{x}^{\alpha} \nabla \nabla \cdot \omega, \nabla \partial_{x}^{\alpha} \sigma\right\rangle \\
& -\gamma_{2}\left\langle\partial_{x}^{\alpha} \nabla z, \nabla \partial_{x}^{\alpha} \sigma\right\rangle+\left\langle\partial_{x}^{\alpha} S_{2}, \nabla \partial_{x}^{\alpha} \sigma\right\rangle \\
& -\gamma_{1}\left\langle\partial_{x}^{\alpha} \omega, \nabla \partial_{x}^{\alpha} \nabla \cdot \omega\right\rangle+\left\langle\partial_{x}^{\alpha} \omega, \nabla \partial_{x}^{\alpha} S_{1}\right\rangle .
\end{aligned}
$$

Then by using the Young inequality and integration by parts, it follows from the above equality that

$$
\begin{aligned}
& \frac{3 \gamma_{1}}{4}\left\|\nabla \partial_{x}^{\alpha} \sigma\right\|^{2}+\frac{d}{d t}\left\langle\partial_{x}^{\alpha} \omega, \nabla \partial_{x}^{\alpha} \sigma\right\rangle \\
& \leq C\left\|\partial_{x}^{\alpha} \nabla^{2} \omega\right\|^{2}+\left\|\partial_{x}^{\alpha} \nabla \cdot \omega\right\|^{2}+\left\|\partial_{x}^{\alpha} \nabla z\right\|^{2} \\
& \quad+C\left|\left\langle\nabla \partial_{x}^{\alpha} \omega, \partial_{x}^{\alpha} S_{1}\right\rangle\right|+C\left|\left\langle\nabla \partial_{x}^{\alpha} \sigma, \partial_{x}^{\alpha} S_{2}\right\rangle\right| .
\end{aligned}
$$

Similar to the estimates of $I_{1}^{k}(t)$ and $I_{2}^{k}(t)$ in the proof of Lemma 8, we have

$$
\begin{aligned}
& \left|\left\langle\nabla \partial_{x}^{\alpha} \omega, \partial_{x}^{\alpha} S_{1}\right\rangle\right| \leq C\left\|\nabla \partial_{x}^{\alpha} \omega\right\|^{2}+C \delta\left(\|\nabla \sigma\|^{2}+\|\nabla \omega\|_{2}^{2}\right), \\
& \left|\left\langle\nabla \partial_{x}^{\alpha} \sigma, \partial_{x}^{\alpha} S_{2}\right\rangle\right| \leq C \delta\left(\left\|\nabla \partial_{x}^{\alpha} \sigma\right\|^{2}+\|\nabla \sigma\|_{1}^{2}+\|\nabla \omega\|_{2}^{2}\right. \\
& \left.\quad+\|\nabla m\|_{2}^{2}+\|\nabla z\|_{1}^{2}\right) .
\end{aligned}
$$

Putting (63) and (64) into (62) and taking $\delta>0$ sufficiently small yield

$$
\begin{aligned}
& \frac{\gamma_{1}}{2}\left\|\nabla \partial_{x}^{\alpha} \sigma\right\|^{2}+\frac{d}{d t}\left\langle\partial_{x}^{\alpha} \omega, \nabla \partial_{x}^{\alpha} \sigma\right\rangle \\
& \leq C\left(\left\|\partial_{x}^{\alpha} \nabla \omega\right\|_{1}^{2}+\left\|\partial_{x}^{\alpha} \nabla z\right\|^{2}\right) \\
& \quad+C \delta\left(\|\nabla \sigma\|_{1}^{2}+\|\nabla \omega\|_{2}^{2}+\|\nabla m\|_{2}^{2}+\|\nabla z\|_{1}^{2}\right) .
\end{aligned}
$$

Thus, the proof of Lemma 9 is finished.

Let $0 \leq l \leq 1$. By summing up (36) from $k=l$ to 2 , since $\delta$ is small, there exists a constant $C_{1}>0$ such that

$$
\begin{aligned}
& \frac{d}{d t} \sum_{l \leq k \leq 2}\left\|\nabla^{k}(\sigma, \omega, m, z)(t)\right\|^{2} \\
& \quad+\sum_{l \leq k \leq 2}\left\|\nabla^{k+1}(\omega, m, z)(t)\right\|^{2} \leq C_{1} \delta\|\nabla \sigma(t)\|_{1}^{2} .
\end{aligned}
$$

Summing up (57) for from $k=l$ to 1 , since $\delta$ is small, we have

$$
\begin{aligned}
& \frac{d}{d t} \sum_{l \leq k \leq 1}\left\langle\nabla^{k} \omega(t), \nabla^{k} \nabla \sigma(t)\right\rangle+C_{2} \sum_{l \leq k \leq 1}\left\|\nabla^{k} \nabla \sigma(t)\right\|^{2} \\
& \quad \leq C\left\{\sum_{l \leq k \leq 2}\left\|\nabla^{k+1} \omega(t)\right\|^{2}+\sum_{l \leq k \leq 1}\left\|\nabla^{k+1} z(t)\right\|^{2}\right. \\
& \left.\quad+\delta\|\nabla(\sigma, \omega, m, z)(t)\|^{2}\right\},
\end{aligned}
$$

where $C_{2}>0$ is a constant. Multiplying (67) by $2 C_{1} \delta / C_{2}<$ $1 / 2$, adding it with (66), taking $l=0$, and using the smallness of $\delta$, we have

$$
\begin{aligned}
& \frac{d}{d t}\left\{\sum_{0 \leq k \leq 2}\left\|\nabla^{k}(\sigma, \omega, m, z)(t)\right\|^{2}\right. \\
& \left.+2 \frac{C_{1} \delta}{C_{2}} \sum_{0 \leq k \leq 1}\left\langle\nabla^{k} \omega(t), \nabla^{k} \nabla \sigma(t)\right\rangle\right\} \\
& +C\left\{\sum_{0 \leq k \leq 1}\left\|\nabla^{k+1} \sigma(t)\right\|^{2}\right. \\
& \left.+\sum_{0 \leq k \leq 2}\left\|\nabla^{k+1}(\omega, m, z)(t)\right\|^{2}\right\} \leq 0
\end{aligned}
$$

Integrating (68) with respect to $t$ and using the smallness of $\delta$, we get (34). The proof of Proposition 6 is complete. 


\section{Decay Rates}

In this section, we get decay rates of solutions to problem (25). Let $\mathbb{A}$ be the following $8 \times 8$ matrix of the differential operators of the form

$$
\mathbb{A}=\left(\begin{array}{cccc}
0 & \gamma_{1} d i v & 0 & 0 \\
\gamma_{1} \nabla & -\mu_{1} \Delta-\mu_{2} \nabla d i v & 0 & \gamma_{2} \nabla \\
0 & 0 & -\nu \Delta & 0 \\
0 & \gamma_{2} d i v & 0 & -\kappa_{1} \Delta
\end{array}\right) \text {. }
$$

If we set

$$
\begin{aligned}
\widetilde{U} & =(\widetilde{\sigma}, \widetilde{\omega}, \widetilde{m}, \widetilde{z})^{t}, \\
U(0) & =\left(\sigma_{0}, \omega_{0}, m_{0}, z_{0}\right)^{t},
\end{aligned}
$$

the linearized problem of (25) is written as

$$
\begin{aligned}
\widetilde{U}_{t}+\mathbb{A} \widetilde{U} & =0, \quad \text { for } t>0, \\
\left.\widetilde{U}\right|_{t=0} & =U(0) .
\end{aligned}
$$

By taking the Fourier transform of (71) with respect to the $x$-variable and solving the ordinary differential equation with respect to $t$, we have

$$
\widetilde{U}(t)=E(t) U(0), \quad t \geq 0,
$$

where $E(t)=e^{-t \mathbb{A}}(t \geq 0)$ is the semigroup generated by the linear operator $-\mathbb{A}$. We define $E(t) f=\mathscr{F}^{-1}\left(e^{-t \widehat{\mathbb{A}}} \xi \widehat{f}(\xi, t)\right)$ for function $f(x, t)$ with

$$
\widehat{\mathbb{A}}(\xi)
$$

$$
\begin{aligned}
& =\left(\begin{array}{cccc}
0 & \sqrt{-1} \gamma_{1} \xi^{t} & 0 & 0 \\
\sqrt{-1} \gamma_{1} \xi & \mu_{1}|\xi|^{2}+\mu_{2} \xi \xi^{t} & 0 & \sqrt{-1} \gamma_{2} \xi \\
0 & 0 & \nu|\xi|^{2} & 0 \\
0 & \sqrt{-1} \gamma_{2} \xi^{t} & 0 & \bar{\kappa}|\xi|^{2}
\end{array}\right), \\
& \text { for } \xi=\left(\begin{array}{l}
\xi_{1} \\
\xi_{2} \\
\xi_{3} \\
\xi_{4}
\end{array}\right)
\end{aligned}
$$

In what following, we denote the solutions to the nonlinear problem (25) by

$$
U(t)=(\sigma(t), \omega(t), m(t), z(t))^{t} .
$$

Then (28) can be rewritten as follows:

$$
\begin{aligned}
U_{t}+\mathbb{A} U & =S(U), \quad \text { for } t>0, \\
\left.U\right|_{t=0} & =U(0),
\end{aligned}
$$

where $S(U) \triangleq\left(S_{1}, S_{2}, S_{3}, S_{4}\right)^{t}$. By using the Duhamel principle, we have

$$
U(t)=E(t) U_{0}+\int_{0}^{t} E(t-\tau) S(U)(\tau) d \tau, \quad t \geq 0
$$

Let $\varphi_{0}(\xi)$ be a function in $C_{0}^{\infty}\left(\mathbb{R}^{3}\right)$ such that

$$
\varphi_{0}(\xi)= \begin{cases}1, & \text { for }|\xi| \leq \frac{R}{2} \\ 0, & \text { for }|\xi| \geq R\end{cases}
$$

where $R$ is some fixed positive constant. Based on the Fourier transform and (77), we can define the low frequency and the high frequency decomposition $\left(f_{L}(x), f_{H}(x)\right)$ for $f(x)$ as follows:

$$
\begin{aligned}
f_{L} & =\mathscr{F}^{-1}\left(\varphi_{0}(\xi) \widehat{f}(\xi)\right), \\
f_{H} & =f-f_{L}
\end{aligned}
$$

By noticing definitions (77) and (78), using the Plancherel theorem, we can obtain the following estimates:

$$
\begin{aligned}
\|\nabla f\| & \leq C\left(\left\|\nabla f_{L}\right\|+\left\|\nabla f_{H}\right\|\right), \\
C\left\|\nabla f_{H}\right\| & \leq\left\|\nabla^{k} f_{H}\right\|, \\
C\left\|\nabla^{k} f_{H}\right\| & \leq\left\|\nabla^{k} f\right\|,
\end{aligned}
$$

for $k \geq 1$

Denote $U_{L}(t) \triangleq \mathscr{F}^{-1}\left(\varphi_{0}(\xi) \widehat{U}(\xi, t)\right)$ and $E_{L}(t) f \triangleq$ $\mathscr{F}^{-1}\left(\varphi_{0}(\xi) e^{-t \widehat{\mathrm{A}}(\xi)} \widehat{f}(\xi, t)\right)$ as the definition (78).

Then from (76), we have

$$
U_{L}(t)=E_{L}(t) U_{0}+\int_{0}^{t} E_{L}(t-\tau) S(U)(\tau) d \tau
$$

To estimate $U_{L}(t)$, we need the $L^{p}-L^{q}$ type of the time decay estimates on the low-frequency part of the semigroup $E(t)$. Notice that the first, the second, and the fourth equations in (71) have been studied in [29]. The magnetic field $H(t, x)$ satisfies the heat equation, which can be solved in Fourier space, $\widehat{H}=\exp \left(-|\xi|^{2} t\right) \widehat{H}_{0}$, whose decay estimate has been proved in many papers, for instance, $[26,27]$. Then the semigroup $E(t)$ has the following properties on the decay in time.

Lemma 10 (see [29]). Let $k \geq 0$ be integers and $1 \leq p \leq 2 \leq$ $q \leq \infty$, then for any $t>0$, it holds that

$$
\begin{aligned}
& \left\|\nabla^{k} E_{L}(t) f\right\| L^{q} \\
& \quad \leq C(k, p, q)(1+t)^{-(3 / 2)(1 / p-1 / q)-k / 2}\|f\|_{L^{p}} .
\end{aligned}
$$

4.1. Decay Estimates of the Low-Frequency Part. In this subsection, we have the decay estimates of $U_{L}(t)$ with the $L^{p}-L^{q}$ type estimate (82) as follows. 
Lemma 11. For $k \geq 0$, it holds that

$$
\begin{aligned}
& \left\|\nabla^{k} U_{L}(t)\right\| \\
& \leq \quad C(1+t)^{-(3 / 2)(1 / p-1 / 2)-k / 2} K_{0} \\
& \quad+C(\delta+\varepsilon) \int_{0}^{t}(1+t-\tau)^{-3 / 4-k / 2}\|\nabla U(\tau)\|_{1} d \tau,
\end{aligned}
$$

where $K_{0}=\left\|U_{0}\right\|_{L^{p}}$ is finite.

Proof. From (81) and Lemma 10, we have

$$
\begin{aligned}
& \left\|\nabla^{k} U_{L}(t)\right\| \\
& \leq C(1+t)^{-(3 / 2)(1 / p-1 / 2)-k / 2}\left\|U_{0}\right\|_{L^{p}} \\
& \quad+C \int_{0}^{t}(1+t-\tau)^{3 / 4-k / 2}\|S(U)(\tau)\|_{L^{1}} d \tau .
\end{aligned}
$$

For the terms including $\bar{\rho}$ on the RHS of (84), by using the Hölder inequality, (12), and the Hardy inequality, we get

$$
\begin{aligned}
& \|\nabla \bar{\rho} \cdot \omega\|_{L^{1}} \leq\|(1+|x|) \nabla \bar{\rho}\|\left\|\frac{\omega}{1+|x|}\right\| \leq C \varepsilon\|\nabla \omega\|, \\
& \|\bar{\rho} \nabla \cdot \omega\|_{L^{1}} \leq\|\bar{\rho}\|\|\nabla \cdot \omega\| \leq C \varepsilon\|\nabla \omega\| .
\end{aligned}
$$

Thus, it holds that

$$
\|\nabla \cdot(\bar{\rho} \omega)\|_{L^{1}} \leq C \varepsilon\|\nabla \omega\|
$$

Similarly, the above inequalities give that

$$
\begin{array}{r}
\left\|\bar{\rho}\left(m \cdot \nabla m-\frac{1}{2} \nabla|m|^{2}\right)\right\|_{L^{1}} \leq \varepsilon\|\nabla m\|_{1}, \\
\|\nabla(\bar{\rho} \sigma)\|_{L^{1}} \leq C \varepsilon\|\nabla \sigma\|_{1}, \\
\|\nabla(\bar{\rho} z)\|_{L^{1}} \leq C \varepsilon\|\nabla z\|_{1}, \\
\|\bar{\rho} \nabla \nabla \cdot \omega\|_{L^{1}} \leq C \varepsilon\left\|\nabla^{2} \omega\right\|, \\
\|\bar{\rho} \Delta z\|_{L^{1}} \leq C \varepsilon\left\|\nabla^{2} z\right\| .
\end{array}
$$

The other terms can be estimated easily. Then, from (38) and the a priori assumption (33), we have

$$
\begin{aligned}
& \|S(U)(t)\|_{L^{1}} \leq C\left(\|(\sigma, \omega, m)\|_{1}\|\nabla(\sigma, \omega, m, z)\|_{1}\right. \\
& \quad+\|\nabla \cdot(\bar{\rho} \omega)\|_{L^{1}}+\|\nabla(\bar{\rho} \sigma)\|_{L^{1}} \\
& \quad+\left\|\bar{\rho}\left(m \cdot \nabla m-\frac{1}{2} \nabla|m|^{2}\right)\right\|_{L^{1}}+\|\nabla(\bar{\rho} z)\|_{L^{1}} \\
& \left.\quad+\left\|\bar{\rho} \nabla^{2}(\omega, z)\right\|_{L^{1}}\right) \leq C(\delta+\varepsilon)\|\nabla(\sigma, \omega, m, z)\|_{1} .
\end{aligned}
$$

4.2. Decay Rates for the Nonlinear Problem. In this subsection, from the a priori estimates and the low frequency and high frequency decomposition (78), we derive a new Lyapunov-type inequality, firstly. From (66) and (67), by taking $l=1$, similar to the estimate (68), we get

$$
\begin{aligned}
& \frac{d \mathscr{H}(t)}{d t}+\left\|\nabla^{2}(\sigma, \omega, m, z)(t)\right\|^{2}+\left\|\nabla^{2}(\omega, m, z)(t)\right\|_{1}^{2} \\
& \leq C \delta\|\nabla(\sigma, \omega, m, z)(t)\|^{2},
\end{aligned}
$$

where $\mathscr{H}(t) \sum_{1 \leq k \leq 2}\left\|\nabla^{k}(\sigma, \omega, m, z)(t)\right\|^{2}+\left\langle\nabla \omega(t), \nabla^{2} \sigma(t)\right\rangle$ is an energy functional which is equivalent to $\|\nabla(\sigma, \omega, z)(t)\|_{1}^{2}$; that means, there exists a positive constant $C_{3}$ such that

$$
\begin{gathered}
\frac{1}{C_{3}}\|\nabla(\sigma, \omega, m, z)(t)\|_{1}^{2} \leq \mathscr{H}(t) \\
\quad \leq C_{3}\|\nabla(\sigma, \omega, m, z)(t)\|_{1}^{2} .
\end{gathered}
$$

By using (79) and (80), from (89), there exists a constant $C_{4}>$ 0 such that

$$
\begin{aligned}
& \frac{d \mathscr{H}(t)}{d} t+C_{4}\left\|\nabla\left(\sigma_{H}, \omega_{H}, m_{H}, z_{H}\right)(t)\right\|^{2} \\
& \quad+\frac{1}{2}\left\|\nabla^{2}(\sigma, \omega, m, z)(t)\right\|^{2}+\frac{1}{2}\left\|\nabla^{2}(\omega, m, z)(t)\right\|_{1}^{2} \\
& \quad \leq C \delta\left\{\left\|\nabla\left(\sigma_{L}, \omega_{L}, m_{L}, z_{L}\right)(t)\right\|^{2}\right. \\
& \left.\quad+\left\|\nabla\left(\sigma_{H}, \omega_{H}, m_{H}, z_{H}\right)(t)\right\|^{2}\right\} .
\end{aligned}
$$

It follows from (91) and the smallness of $\delta$ that

$$
\begin{aligned}
& \frac{d \mathscr{H}(t)}{d t}+C_{5}\left\|\nabla\left(\sigma_{H}, \omega_{H}, m_{H}, z_{H}\right)(t)\right\|^{2} \\
& \quad+\frac{1}{2}\left\|\nabla^{2}(\sigma, \omega, m, z)(t)\right\|^{2}+\frac{1}{2}\left\|\nabla^{2}(\omega, m, z)(t)\right\|_{1}^{2} \\
& \leq C \delta\left\|\nabla\left(\sigma_{L}, \omega_{L}, m_{L}, z_{L}\right)(t)\right\|^{2},
\end{aligned}
$$

where $C_{5}>0$ is a constant. Adding $C_{5}\left\|\nabla\left(\sigma_{L}, \omega_{L}, m_{L}, z_{L}\right)(t)\right\|^{2}$ to the both sides of (92), with the definition of $\mathscr{H}(t)$, there exists a constant $D>0$ such that

$$
\frac{d \mathscr{H}(t)}{d t}+D \mathscr{H}(t) \leq C\left\|\nabla U_{L}(t)\right\|^{2} .
$$

To prove the decay estimates stated in Theorem 1, we first define

$$
M(t)=\sup _{0 \leq \tau \leq t}(1+\tau)^{3(1 / p-1 / 2)+1} \mathscr{H}(\tau)
$$

$M(t)$ is obviously nondecreasing and

$$
\begin{aligned}
\|\nabla U(\tau)\|_{1} & \leq C \sqrt{\mathscr{H}(\tau)} \\
& \leq C(1+\tau)^{-(3 / 2)(1 / p-1 / 2)-1 / 2} \sqrt{M(t)}, \\
& 0 \leq \tau \leq t .
\end{aligned}
$$


Then, from (83), by using Lemma 4 and (95), we obtain

$$
\begin{aligned}
& \left\|\nabla U_{L}(t)\right\| \leq C K_{0}(1+t)^{-(3 / 2)(1 / p-1 / 2)-1 / 2}+C(\delta+\varepsilon) \\
& \quad \cdot \int_{0}^{t}(1+t-\tau)^{-5 / 4} \\
& \quad \cdot(1+\tau)^{-(3 / 2)(1 / p-1 / 2)-1 / 2} d \tau \sqrt{M(t)} \\
& \quad \leq C(1+t)^{-(3 / 2)(1 / p-1 / 2)-1 / 2}\left(K_{0}+(\delta+\varepsilon) \sqrt{M(t)}\right) .
\end{aligned}
$$

By Gronwall's inequality and (96), from (93), hence

$$
\begin{gathered}
\mathscr{H}(t) \leq \mathscr{H}(0) e^{-D t}+C_{2} \int_{0}^{t} e^{-D(t-\tau)}\left\|\nabla U_{L}(\tau)\right\|^{2} d \tau \leq \mathscr{H}(0) e^{-D t} \\
+C \int_{0}^{t} e^{-D(t-\tau)}(1+\tau)^{-(3 / 2)(1 / p-1 / 2)-1} d \tau\left(K_{0}^{2}+(\delta+\varepsilon)^{2} M(t)\right) \\
\leq(1+t)^{-(3 / 2)(1 / p-1 / 2)-1}\left(\mathscr{H}(0)+K_{0}^{2}+(\delta+\varepsilon)^{2} M(t)\right),
\end{gathered}
$$

where $\mathscr{H}(0)$ is equivalent to $\left\|\nabla\left(\sigma_{0}, \omega_{0}, m_{0}, z_{0}\right)\right\|_{1}^{2}$.

In terms of the definition of $M(t)$, it follows from (97) that

$$
M(t) \leq C\left(\mathscr{H}(0)+K_{0}^{2}\right)+C(\delta+\varepsilon)^{2} M(t),
$$

which implies that if $\delta$ and $\varepsilon$ are sufficiently small then

$$
M(t) \leq C\left(\mathscr{H}(0)+K_{0}^{2}\right) .
$$

By applying the definition of $\mathscr{H}(t)$ and $M(t)$, we have

$$
\|\nabla(\sigma, \omega, m, z)(t)\|_{1} \leq C(1+t)^{-(3 / 2)(1 / p-1 / 2)-1 / 2} .
$$

Then, we have (9).

Then, we define the temporal energy functional

$$
\begin{aligned}
\mathscr{H}_{1}(t)= & \|(\sigma, \omega, m, z)(t)\|_{2}^{2} \\
& +\frac{2 C_{1} \delta}{C_{2}} \sum_{0 \leq k \leq 2}\left\langle\nabla^{k} \sigma(t), \nabla^{k} \nabla \omega(t)\right\rangle,
\end{aligned}
$$

where $\mathscr{H}_{1}(t)$ is equivalent to $\|(\sigma, \omega, m, z)(t)\|_{2}^{2}$, for the positive constants $\delta$ can be sufficiently small. Then, from (68), we have

$$
\begin{aligned}
& \frac{d \mathscr{H}_{1}(t)}{d t}+\|\nabla(\sigma, \omega, m, z)(t)\|^{2}+\|\nabla(\omega, m, z)(t)\|_{1}^{2} \\
& \quad \leq 0 .
\end{aligned}
$$

Similar to the estimate (93), from (102), there exists a positive constant $D_{1}$ such that

$$
\frac{d \mathscr{H}_{1}(t)}{d t}+D_{1} \mathscr{H}_{1}(t) \leq C\left\|U_{L}(t)\right\|^{2}
$$

Taking $k=0$ in (83) and using (100), for $1 \leq p \leq 2$, we obtain

$$
\begin{aligned}
& \left\|U_{L}(t)\right\| \leq C(1+t)^{-(3 / 2)(1 / p-1 / 2)} K_{0}+C(\delta+\varepsilon) \\
& \cdot \int_{0}^{t}(1+t-\tau)^{-3 / 4}(1+\tau)^{-(3 / 2)(1 / p-1 / 2)-1 / 2} d \tau \\
& \quad \leq C(1+t)^{-(3 / 2)(1 / p-1 / 2)}\left(K_{0}+C\right),
\end{aligned}
$$

where let $r_{1}=3 / 4, r_{2}=(3 / 2)(1 / p-1 / 2)+1 / 2$ and $\eta=1 / 4$ in Lemma 4. get

From (103), by using Gronwall's inequality and (104), we

$$
\begin{aligned}
\mathscr{H}_{1}(t) & \leq \mathscr{H}_{1}(0) e^{-D_{1} t}+C \int_{0}^{t} e^{-D_{1}(t-\tau)}\left\|U_{L}(\tau)\right\|^{2} d \tau \\
\leq & \mathscr{H}(0) e^{-D_{1} t} \\
& +\left(C+C K_{0}^{2}\right) \int_{0}^{t} e^{-D_{1}(t-\tau)}(1+\tau)^{-3(1 / p-1 / 2)} d \tau \\
\leq & C(1+t)^{-3(1 / p-1 / 2)}\left(\mathscr{H}_{1}(0)+C+K_{0}^{2}\right),
\end{aligned}
$$

where $\mathscr{H}_{1}(0)$ is equivalent to $\left\|\left(\sigma_{0}, \omega_{0}, m_{0}, z_{0}\right)\right\|_{2}^{2}$. Then, it follows from the definition of $\mathscr{H}_{1}(t)$ that

$$
\|(\sigma, \omega, m, z)(t)\| \leq C(1+t)^{-(3 / 2)(1 / p-1 / 2)} .
$$

Finally, by the interpolation, for any $2 \leq q \leq 6$, plugging (106) into (100) yields

$$
\begin{aligned}
\|U(t)\|_{L^{q}} & \leq\|U(t)\|_{L^{6}}^{\eta_{0}}\|U(t)\|^{1-\eta_{0}} \\
& \leq C\|\nabla U(t)\|^{\eta_{0}}\|U(t)\|^{1-\eta_{0}} \\
& \leq C(1+t)^{-(3 / 2)(1 / p-1 / q)},
\end{aligned}
$$

where $\eta_{0}=3(q-2) / 2 q$. Then, we get (10).

On the other hand, by using (25), Lemma 3, (9), and (12), we have

$$
\begin{aligned}
& \left\|\partial_{t}(\sigma, \omega, m, z)(t)\right\|_{L^{2}} \leq C\|\nabla \cdot \omega(t)\|_{L^{2}}+\left\|S_{1}(t)\right\|_{L^{2}} \\
& \quad+C\|\nabla \sigma(t)\|_{L^{2}}+C\|\nabla z(t)\|_{L^{2}}+C\|\Delta \omega(t)\|_{L^{2}} \\
& \quad+C\|\nabla \nabla \cdot \omega(t)\|_{L^{2}}+\left\|S_{2}(t)\right\|_{L^{2}}+C\|\Delta m(t)\|_{L^{2}} \\
& \quad+\left\|S_{3}(t)\right\|_{L^{2}}+C\|\Delta z(t)\|_{L^{2}}+\left\|S_{4}(t)\right\|_{L^{2}} \\
& \quad \leq C\left\{\|\nabla \sigma(t)\|_{L^{2}}+\|\nabla \omega(t)\|_{H^{1}}+\|\nabla m(t)\|_{H^{1}}\right. \\
& \left.\quad+\|\nabla z(t)\|_{H^{1}}\right\} \leq C(1+t)^{-(3 / 2)(1 / p-1 / 2)-1 / 2}
\end{aligned}
$$

for any $0 \leq t \leq T$.

This completes the proof of Theorem 1 .

\section{Data Availability}

The data used to support the findings of this study are available from the corresponding author upon request.

\section{Conflicts of Interest}

The author declares that they have no conflicts of interest.

\section{References}

[1] A. Matsumura and T. Nishida, "Initial boundary value problems for the equations of motion of compressible viscous and heat conductive fluids," Communications in Mathematical Physics, vol. 89, no. 4, pp. 445-464, 1983. 
[2] G.-Q. Chen and D. Wang, "Global solutions of nonlinear magnetohydrodynamics with large initial data," Journal of Differential Equations, vol. 182, no. 2, pp. 344-376, 2002.

[3] G.-Q. Chen and D. Wang, "Existence and continuous dependence of large solutions for the magnetohydrodynamics equations," Nonlinear Analysis-Theory Methods \& Applications, vol. 54, no. 4, pp. 608-632, 2003.

[4] Q. Chen and Z. Tan, "Global existence and convergence rates of smooth solutions for the compressible magnetohydrodynamic equations," Nonlinear Analysis, vol. 72, no. 12, pp. 4438-4451, 2010.

[5] C. Dou and Q. Ju, "Low Mach number limit for the compressible magnetohydrodynamic equations in a bounded domain for all time," Communications in Mathematical Sciences, vol. 12, no. 4, pp. 661-679, 2014.

[6] J. Fan, S. Jiang, and G. Nakamura, "Vanishing shear viscosity limit in the magnetohydrodynamic equations," Communications in Mathematical Physics, vol. 270, no. 3, pp. 691-708, 2007.

[7] E. Feireisl, A. Novotný, and Y. Sun, "Dissipative solutions and the incompressible inviscid limits of the compressible magnetohydrodynamic system in unbounded domains," Discrete and Continuous Dynamical Systems, vol. 34, no. 1, pp. 121-143, 2014.

[8] D. Hoff and E. Tsyganov, "Uniqueness and continuous dependence of weak solutions in compressible magnetohydrodynamics," Journal of Differential Equations, vol. 56, pp. 215-254, 2005.

[9] X. Hu and D. Wang, "Compactness of weak solutions to the three-dimensional compressible magnetohydrodynamic equations," Journal of Differential Equations, vol. 245, no. 8, pp. 21762198, 2008.

[10] X. Hu and D. Wang, "Global solutions to the three-dimensional full compressible magnetohydrodynamic flows," Communications in Mathematical Physics, vol. 283, no. 1, pp. 255-284, 2008.

[11] X. Hu and D. Wang, "Global existence and large-time behavior of solutions to the three-dimensional equations of compressible magnetohydrodynamic flows," Archive for Rational Mechanics and Analysis, vol. 197, no. 1, pp. 203-238, 2010.

[12] S. Jiang, Q. Ju, F. Li, and Z. Xin, "Low Mach number limit for the full compressible magnetohydrodynamic equations with general initial data," Advances in Mathematics, vol. 259, pp. 384420, 2014.

[13] F. Li and H. Yu, "Optimal decay rate of classical solutions to the compressible magnetohydrodynamic equations," Proceedings of the Royal Society of Edinburgh, Section: A Mathematics, vol. 141, no. 1, pp. 109-126, 2011.

[14] H.-L. Li, X. Xu, and J. Zhang, "Global classical solutions to $3 \mathrm{D}$ compressible magnetohydrodynamic equations with large oscilla-tions and vacuum," SIAM Journal on Mathematical Analysis, vol. 45, no. 3, pp. 1356-1387, 2013.

[15] X. Li, N. Su, and D. Wang, "Local strong solution to the compressible magnetohydrodynamic flow with large data," Journal of Hyperbolic Differential Equations, vol. 8, no. 3, pp. 415-436, 2011.

[16] Y. Li, "Convergence of the compressible magnetohydrodynamic equations to incompressible magnetohydrodynamic equations," Journal of Differential Equations, vol. 252, no. 3, pp. 2725-2738, 2012.

[17] X. Pu and B. Guo, "Global existence and convergence rates of smooth solutions for the full compressible MHD equations," Nonlinear Analysis-Real World Applications, vol. 64, no. 3, pp. 519-538, 2013.
[18] Z. Tan and H. Wang, "Optimal decay rates of the compressible magnetohydrodynamic equations," Nonlinear Analysis: Real World Applications, vol. 14, no. 1, pp. 188-201, 2013.

[19] Y.-Z. Wang and Y.-X. Wang, "Local existence of strong solutions to the three dimensional compressible MHD equations with partial viscosity," Communications on Pure and Applied Analysis, vol. 12, no. 2, pp. 851-866, 2013.

[20] B. Yuan and X. Zhao, "Blow up of smooth solutions to the full compressible MHD system with compact density," Kinetic and Related Models, vol. 7, no. 3, pp. 195-203, 2014.

[21] A. Matsumura and T. Nishida, "The initial value problem for the equations of motion of compressible viscous and heatconductive fluids," Proceedings of the Japan Academy, Series A: Mathematical Sciences, vol. 55, no. 9, pp. 337-342, 1979.

[22] G. Ponce, "Global existence of small solutions to a class of nonlinear evolution equations," Nonlinear Analysis: Theory, Methods \& Applications, vol. 9, no. 5, pp. 399-418, 1985.

[23] K. Deckelnick, "Decay estimates for the compressible NavierStokes equations in unbounded domains," Mathematische Zeitschrift, vol. 209, no. 1, pp. 115-130, 1992.

[24] R. Duan, S. Ukai, T. Yang, and H. Zhao, "Optimal convergence rates for the compressible Navier-Stokes equations with potential forces," Mathematical Models and Methods in Applied Sciences, vol. 17, no. 5, pp. 737-758, 2007.

[25] W. Wang, "Optimal convergence rates for the strong solutions to the compressible Navier-Stokes equations with potential force," Nonlinear Analysis: Real World Applications, vol. 34, pp. 363378, 2017.

[26] R. Duan, "Global smooth flows for the compressible EulerMaxwell system. The relaxation case," Journal of Hyperbolic Differential Equations, vol. 8, no. 2, pp. 375-413, 2011.

[27] T. C. Sideris, B. Thomases, and D. Wang, "Long time behavior of solutions to the $3 \mathrm{D}$ compressible Euler equations with damping," Communications in Partial Differential Equations, vol. 28, no. 3-4, pp. 795-816, 2003.

[28] R. A. Adams, Sobolev Spaces, Academic Press, New York, NY, USA, 1985.

[29] T. Kobayashi and Y. Shibata, "Decay estimates of solutions for the equations of motion of compressible viscous and heatconductive gases in an exterior domain in $\mathbb{R}^{3}$," Communications in Mathematical Physics, vol. 200, pp. 621-659, 1999. 


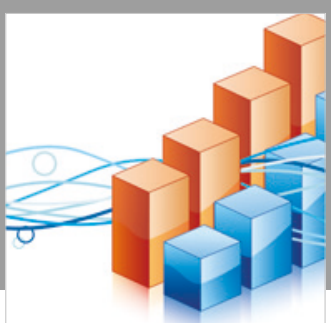

Advances in

Operations Research

\section{-n-m}
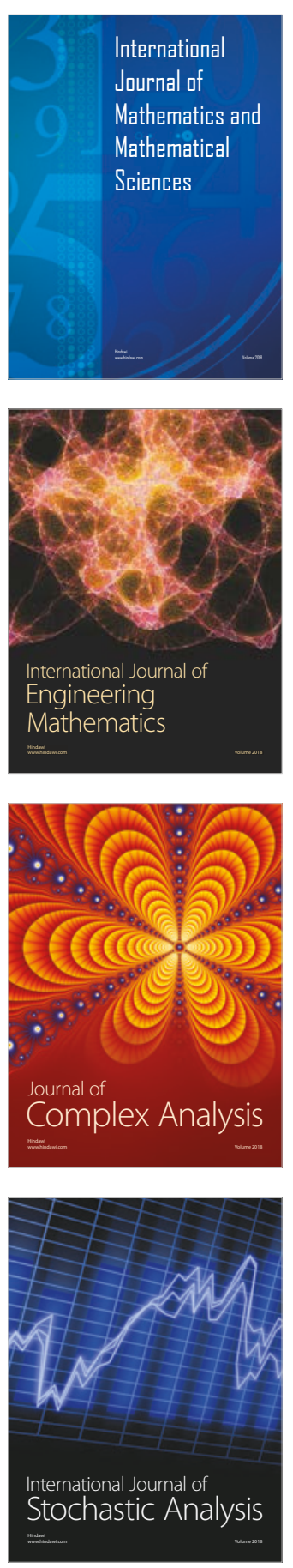
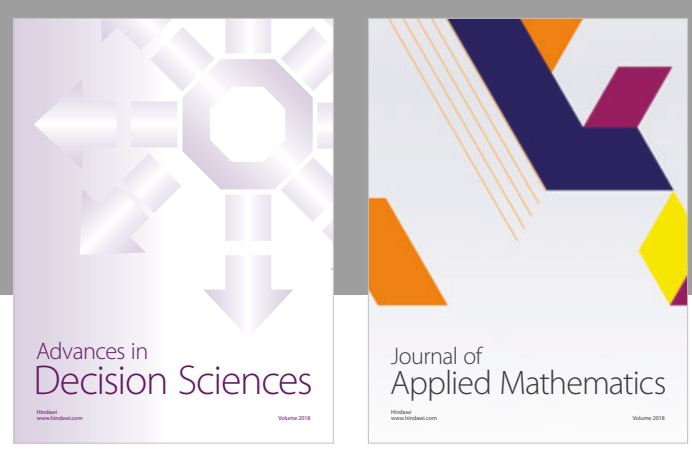

Journal of

Applied Mathematics
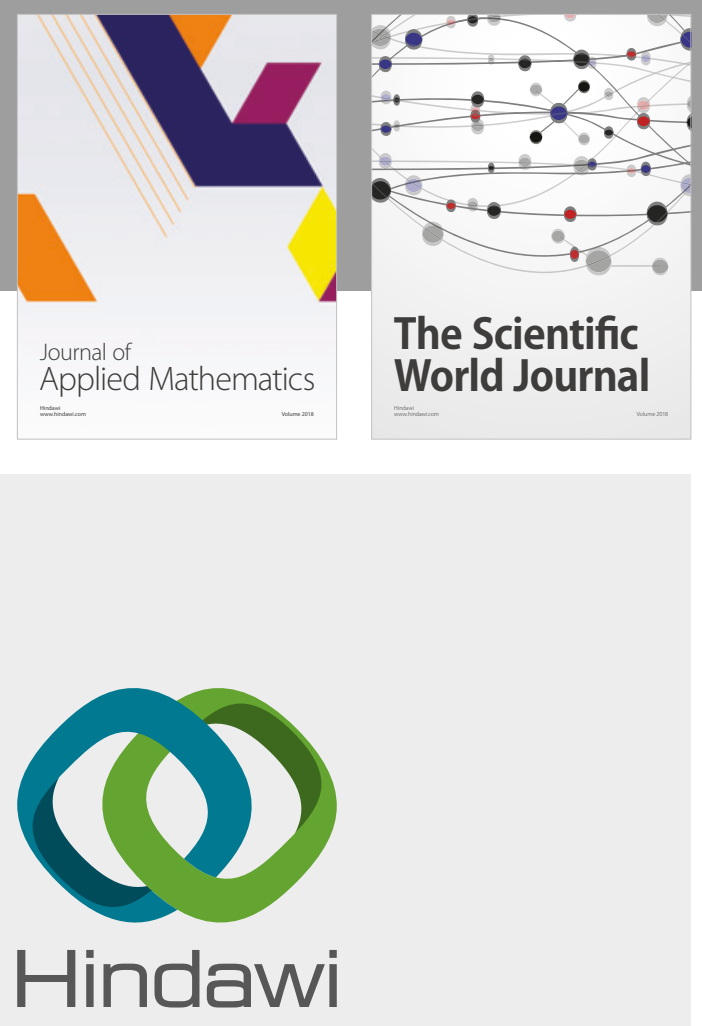

Submit your manuscripts at

www.hindawi.com

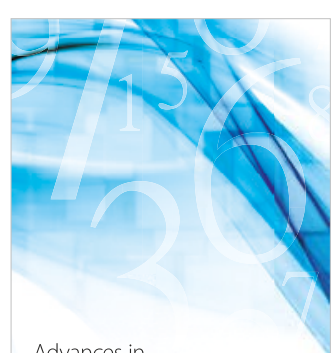

Advances in
Numerical Analysis
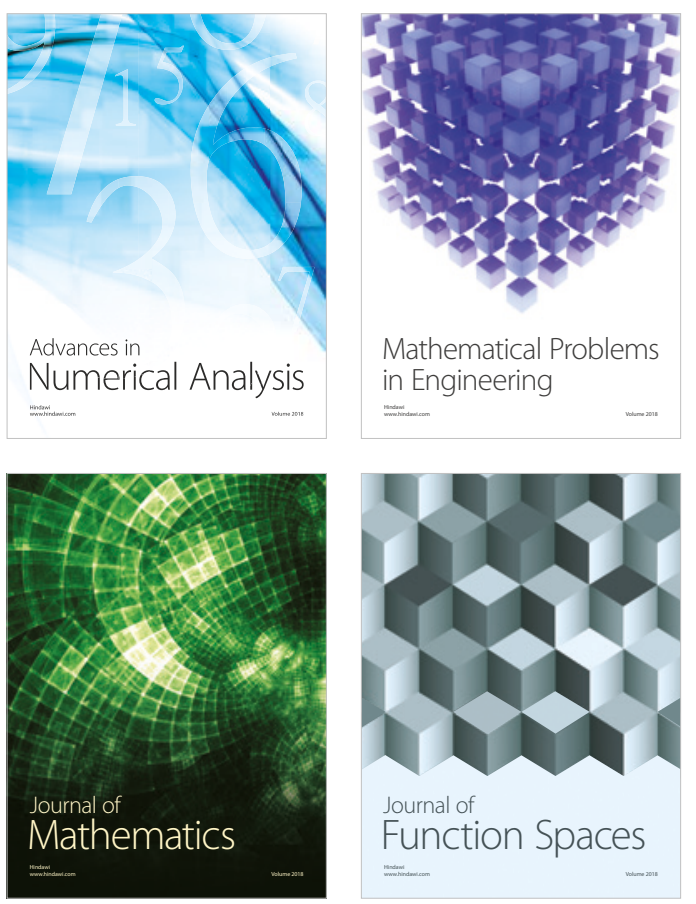

Mathematical Problems in Engineering

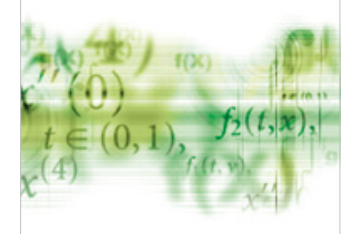

International Journal of

Differential Equations

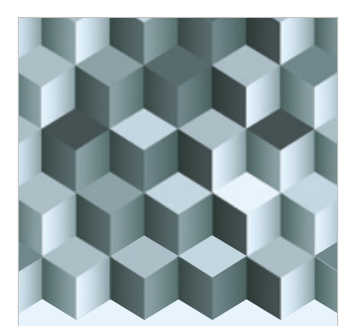

Journal of

Function Spaces

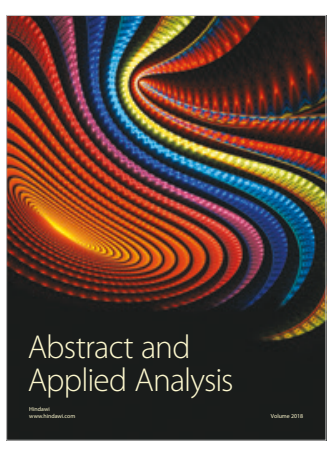

The Scientific

World Journal

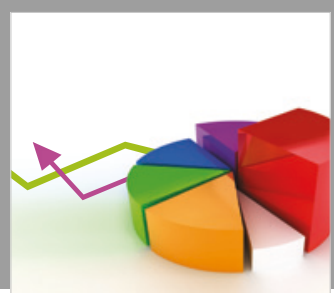

Journal of

Probability and Statistics
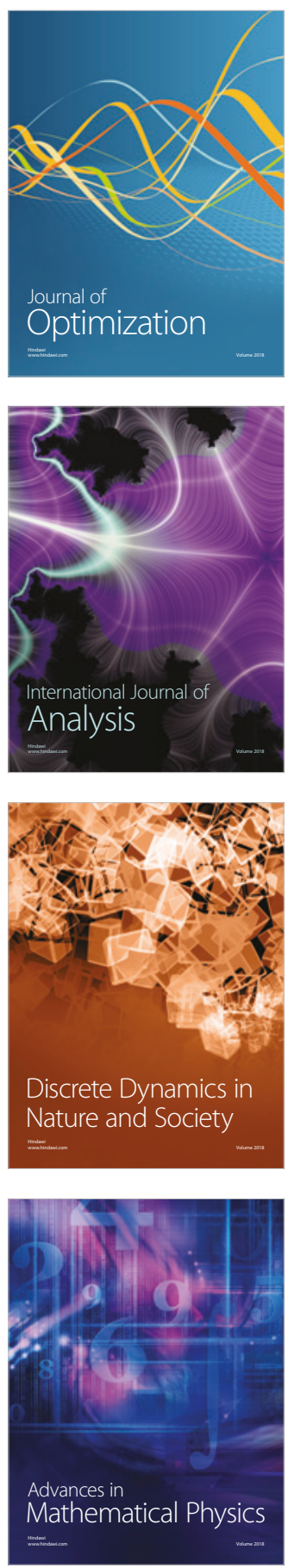